Draft Version July 6, 2018

Preprint typeset using $\mathrm{LATE}_{\mathrm{E}} \mathrm{X}$ style emulateapj v. 5/2/11

\title{
ELECTRON DENSITIES AND ALKALI ATOMS IN EXOPLANET ATMOSPHERES
}

\author{
P. Lavvas ${ }^{1}$, T. Koskinen ${ }^{2}$, And R.V. Yelle ${ }^{2}$ \\ Draft version July 6, 2018
}

\begin{abstract}
We describe a detailed study on the properties of alkali atoms in extrasolar giant planets, and specifically focus on their role in generating the atmospheric free electron densities, as well as their impact on the transit depth observations. We focus our study on the case of HD $209458 \mathrm{~b}$, and we show that photoionization produces a large electron density in the middle atmosphere that is about two orders of magnitude larger than the density anticipated from thermal ionization. Our purely photochemical calculations though result in a much larger transit depth for $\mathrm{K}$ than observed for this planet. This result does not change even if the roles of molecular chemistry and excited state chemistry are considered for the alkali atoms. In contrast, the model results for the case of exoplanet $\mathrm{XO}-2 \mathrm{~b}$ are in good agreement with the available observations. Given these results we discuss other possible scenarios, such as changes in the elemental abundances, changes in the temperature profiles, and the possible presence of clouds, which could potentially explain the observed HD $209458 \mathrm{~b}$ alkali properties. We find that most of these scenarios can not explain the observations, with the exception of a heterogeneous source (i.e. clouds or aerosols) under specific conditions, but we also note the discrepancies among the available observations.

Subject headings: planetary systems planets and satellites: atmospheres, composition, individual (HD 209458 b, XO-2 b)
\end{abstract}

\section{INTRODUCTION}

Alkali atoms and free electrons in planetary atmospheres are strongly related as the low ionization potentials of the former can induce significant populations of the latter. Alkali atoms are expected to be abundant in hot atmospheres where their condensation or loss to other molecular structures will be minimal. In this sense, the hot atmospheres of close-in exoplanets are excellent candidates for the investigation of the alkali-electron relationship, and its subsequent ramifications on the atmospheric electro-dynamics.

Although alkali atoms are now observed in many different exoplanets, we focus our study on the case of HD 209458 b, due to the extended number of observational constraints available for this planet. Observations with the Hubble Space Telescope (HST) at visible wavelengths provided the first detection of an exoplanet atmosphere through the detection of sodium in HD 209458 b (Brown et al. 2001; Charbonneau et al. 2002). Subsequent observations with the same instrument (Ballester et al. 2007; Knutson et al. 2007), reproduced the first detection and verified that the $\mathrm{Na}$ absorption was smaller than anticipated theoretically (Seager and Sasselov 2000; Hubbard et al. 2001; Brown 2001), while potassium and lithium were not detected. Ground based observations in the visible also detected $\mathrm{Na}$ in the atmosphere of HD $209458 \mathrm{~b}$, and verified the lack of K in the measured spectra (Snellen et al. 2008; Jensen et al. 2011).

Based on these measurements, many subsequent studies have focused on their re-analysis and interpretation

\footnotetext{
panayotis.lavvas@univ-reims.fr

${ }^{1}$ GSMA, Université de Reims Champagne Ardenne, CNRS UMR 7331, France

${ }^{2}$ Lunar and Planetary Laboratory, University of Arizona, Tucson, AZ 85719, USA
}

through different scenarios. Barman et al. (2002) suggested that non-LTE effects could be responsible for the decreased Na transit depth observed, while Fortnev et al. (2003), following on the initial scenarios suggested by Charbonneau et al. (2002), investigated the role of ionization and potential cloud formation, and suggested that both processes are important for the absorption in the $\mathrm{Na} D$ line core. Sing et al. (2008b) re-analyzed the HST observations at a higher resolution and suggested that a local temperature minimum must exist close to the mbar pressure level of HD 209458 b, which would allow $\mathrm{Na}$ to condense and reduce its transit depth signature. Vidal-Madiar et al. (2011) used the differential transit depth measurements reported by Sing et al. (2008b) to derive a vertical thermal profile of HD 209458 b's atmosphere, assuming a constant with altitude $\mathrm{Na}$ abundance. The lack of $\mathrm{K}$ in the absorbed spectra, however, has not been investigated in detail before. The commonly anticipated scenarios to explain this lack are a strong photochemical loss (due to the lower ionization of $\mathrm{K}$ relative to $\mathrm{Na}$ ), the screening of the $\mathrm{K}$ signature by a cloud/haze, or the lower than solar abundance of this element in the atmosphere of HD 209458 b (Desert et al. 2008).

In the meanwhile, measurements in other regions of the electromagnetic spectrum, as well as theoretical studies provide more information about the atmospheric properties of HD 209458 b. Transit observations with HST at shorter wavelengths indicate the presence of HI, CII, OI, and SiIII in the thermosphere of this exoplanet (Vidal-Madiar et al. 2003, 2004; Ben-Jaffel and Sona Hosseini 2010; Linsky et al. 2010), which impose important constraints on both the temperature of the thermosphere, as well as the efficiency of condensation of heavy elements in the deeper atmosphere Koskinen et al. 2010; Koskinen et al. 2013a,b). Observations at IR wavelengths during 


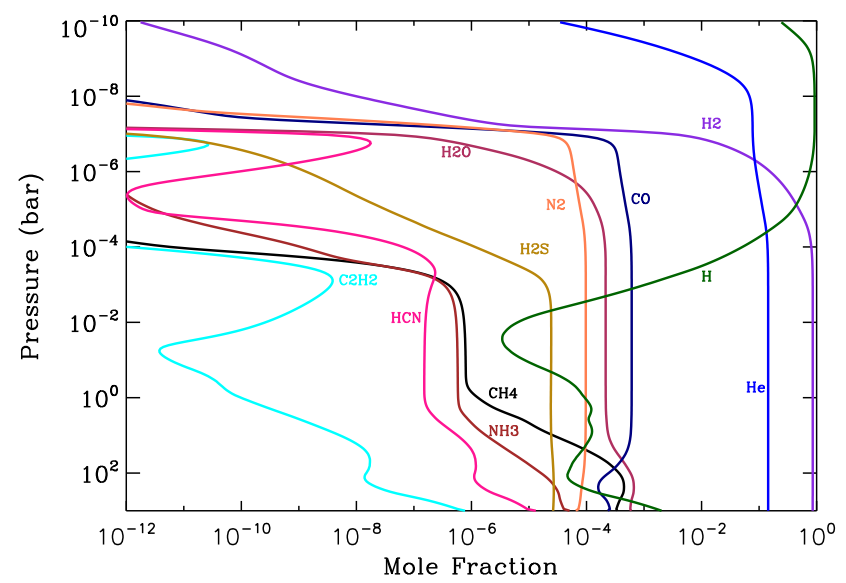

FIG. 1.- Major chemical species for HD209458b calculated by our model.

primary (Beaulieu et al. 2010; Deming et al. 2013) and secondary (Knutson et al. 2008; Swain et al. 2009; Crossfield et al. 2012) eclipse reveal the presence of $\mathrm{H}_{2} \mathrm{O}, \mathrm{CH}_{4}$, and $\mathrm{CO}_{2}$ in the deep atmosphere of $\mathrm{HD}$ $209458 \mathrm{~b}$, and inform us on the temperature conditions of the day side disk and of the terminator. These observations have helped to constrain general circulation models (Showman et al. 2009) and photochemical models (Moses et al. 2011). Such simulations are important because they provide information for the role of dynamics in the redistribution of energy deposited on the day side to the night side, the role of atmospheric mixing, and the possible abundances of the important chemical species that are suggested by the observations.

In light of the new information based on a combination of observations and models, we use a photochemical model to investigate the role of $\mathrm{Na}$ and $\mathrm{K}$ in the atmosphere of HD 209458 b. We focus on the relative abundance of $\mathrm{Na}$ and $\mathrm{K}$ in order to address the surprising lack of a $\mathrm{K}$ signature in the observations. Given the detection of $\mathrm{K}$ in other exoplanet atmospheres such as XO-2 b (Sing et al. 2010), this study is a timely introduction to a comparative investigation into the role of alkali atoms in different exoplanet atmospheres. We first discuss the impact of photochemistry on the vertical profiles of these elements and the produced electron densities, and then proceed to a more thorough study of the role of the excited state chemistry of these elements. Finally, based on the conclusions we derive from our calculations we discuss all the previously suggested scenarios for the interpretation of the observations. The impact of the free electron densities derived here on the atmospheric electro-dynamics is discussed in the accompanying paper (Koskinen et al. 2014).

\section{MODEL DESCRIPTION}

We use a $1 \mathrm{D}$ photochemical model that extends from $10^{3}$ to $10^{-10}$ bar. Our model is based on a previous version applied to Titan's atmosphere (Lavvas et al. 2008), but has been extended to include forward and reverse reactions (with thermochemical parameters from Burcat and Ruscid 2005), and is therefore able to kinetically simulate thermochemical processes for species containing $\mathrm{H}, \mathrm{C}, \mathrm{N}, \mathrm{O}, \mathrm{S}$ and $\mathrm{Si}$. The model solves the

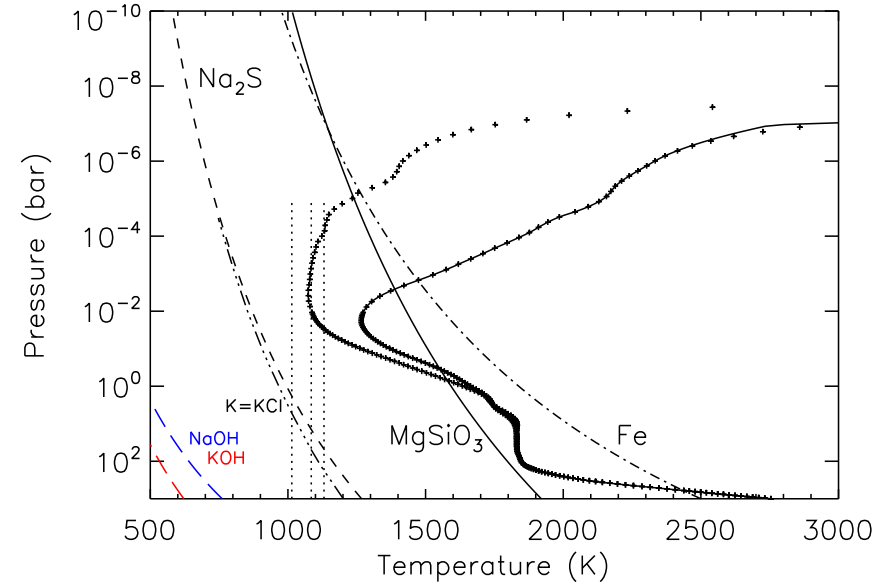

FIG. 2.- Temperature-pressure profiles assumed for day-time (crosses with solid line) and terminator average (crosses) conditions of HD209458b based on Showman et al. (2009). Condensation curves for $\mathrm{Fe}$ (dash-dotted), $\mathrm{MgSiO}_{3}$ (solid), and $\mathrm{Na}_{2} \mathrm{~S}$ (dashed) are also shown. The $\mathrm{K}=\mathrm{KCl}$ curve marks the transition from atomic $\mathrm{K}$ to molecular $\mathrm{KCl}$ under thermochemical equilibrium, while the colored dashed lines show the condensation curves of $\mathrm{NaOH}$ and $\mathrm{KOH}$. The vertical dotted lines present skin temperatures for HD 209458 $\mathrm{b}$ ( $1085 \mathrm{~K}$ for bond albedo of $\mathrm{A}=30 \%$ and $1130 \mathrm{~K}$ for $\mathrm{A}=17 \%$ ) and $\mathrm{XO}-2 \mathrm{~b}(1014 \mathrm{~K}$ for $\mathrm{A}=30 \%)$.

continuity equation for each species taking into consideration photochemical and thermochemical sources and sinks, and the transport of the species due to molecular diffusion and atmospheric mixing (the latter based on an eddy diffusion profile). In total we include $\sim 130$ species involved in $\sim 1600$ reactions. In this study we focus on the processes of ionization and chemistry of alkali species, which have important implications for the interpretation of the available observations. We avoid a full description of the basic $\mathrm{H} / \mathrm{C} / \mathrm{N} / \mathrm{O} / \mathrm{S}$ chemical networks, which have already been well described in previous studies (e.g. Zahnle et al. 2009; Moses et al. 2011; Venot et al. 2012), and focus our study on $\mathrm{Na}$ and $\mathrm{K}$ chemistry. For the case of HD 209458 b our model results for the main atmospheric compounds (Fig. 1) are similar to those of previous studies.

For the atmospheric structure of HD 209458 b we assume the vertical temperature - pressure profiles suggested by general circulation models (Showman et al. 2009) for the disk and terminator conditions (Fig. 2). Above $10^{-6}$ bar we have merged these profiles with the thermospheric temperature profile calculated by Koskinen et al. (2013b). For the atmospheric mixing we consider the eddy diffusion profile derived from GCM for the case of HD 209458 b (Moses et al. 2011). Parmentier et al. (2013) demonstrate that the eddy profile used by Moses et al. (2011) is likely too large for HD 209458 b, therefore we discuss below the effects of different mixing profiles. At the lower boundary we assume solar elemental abundances and thermochemical equilibrium (Lodders 2010b), while at the upper boundary we allow species with mass less than 3 amu to escape with a velocity provided by escape models (Koskinen et al. 2013a, b). The effects of hydrodynamic escape on the heavier species are not included here as we focus on the lower atmosphere. 


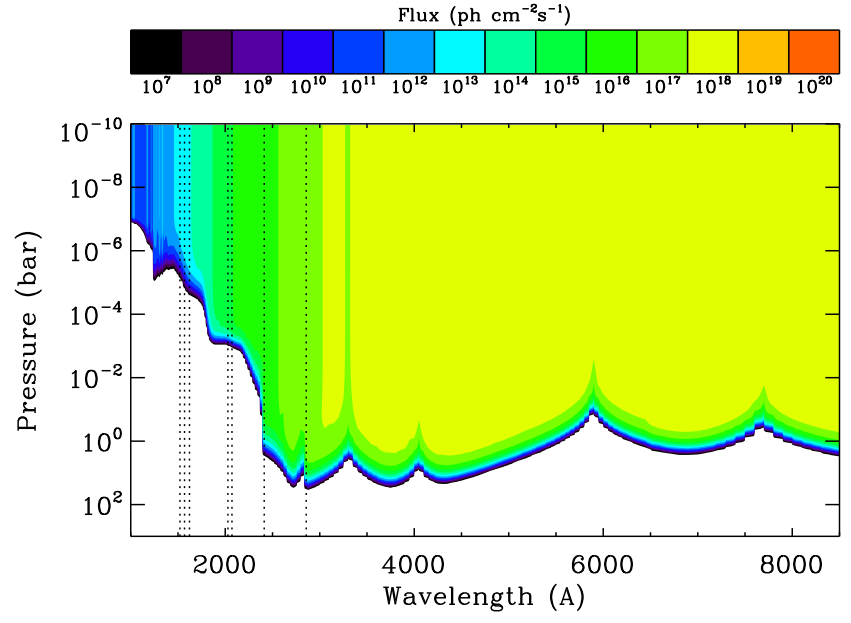

FIG. 3.- Stellar flux penetration in the atmosphere of HD209458b for different wavelengths. The vertical dotted lines show the ionization limits of the heavy atoms included in the calculations (see Table 1), while absorption by $\mathrm{Na}$ and $\mathrm{K}$ resonant lines dominates at longer wavelengths.

We use ionization cross sections from Verner et al. (1996) for $\mathrm{Na}$, and from Sandner et al. (1981) and Zatsarinny and Taval (2010) for K. For Na we use recombination rates from Verner et al. (1996) for the radiative process and from $\mathrm{Su}$ et al. (2001) for the 3-body process. The latter study provides only the low pressure limit rate and we assumed a high pressure limit of $10^{-7} \mathrm{~cm}^{3} \mathrm{~s}^{-1}$. For $\mathrm{K}$ the radiative recombination rate (Landini and Fossi 1991) is very close to the Na radiative rate, and as we did not find any measurements for the 3-body recombination of $\mathrm{K}$, we assumed the same recombination rates as for Na. Thermal ionization rates were calculated on the basis of microscopic reversibility based on the assumed rates of 3-body recombination.

For the stellar spectrum of HD209458 we assume a solar-average spectrum that is representative of $\mathrm{G}$ type stars like HD 209458. Atmospheric attenuation includes opacities from all optically significant species included in the model, and Rayleigh scattering by $\mathrm{H}_{2}$. Fig. 3 presents the attenuated radiation field at different pressure regions of the atmosphere at UV and visible wavelengths. At the ionization limits of $\mathrm{Na}$ and $\mathrm{K}$ (see Table 1 and vertical lines in Fig. 31) significant photon fluxes persist to the deep atmosphere and can induce large ionization rates as we describe below.

\section{RESULTS}

We discuss here the ion and electron densities, resulting from the photoionization of the alkali atoms, and compare our calculated transit depth spectrum, based on the calculated mixing ratio profiles, with the available observations for HD 209458 b. We also discuss possible contributions from the ionization of other atomic compounds.

\subsection{Ions \& Electrons}

Due to their different ionization potentials, ionization of each atom is initiated at a different pressure level in the atmosphere. In the upper atmosphere we consider ionization of $\mathrm{H}, \mathrm{He}, \mathrm{C}, \mathrm{N}$, and $\mathrm{O}$, the ion-chemistry of which we have already discussed in Koskinen et al. (2013a). These
TABLE 1

Properties of ATOMS CONTRIBUTING TO IONIZATION IN THE LOWER ATMOSPhERE. ABUndances ARE GIVEN IN TERMS OF A MiXing RATIO TO $\mathrm{H}_{2}$ CONSIDERING a He MIXING RATIO OF 0.144. FOR THE SPECIAL CASE WHERE WE CONSIDER THE PRESENCE OF OTHER HEAVY ATOMS THAN NA AND K (SECTION 3.3), AT THE LOWER BOUNDARY ALUMINUM IS IN THE FORM OF ALOH AND

AlH, while silicon forms SiO And $\mathrm{SiH}_{4}$. All other elements ARE DOMINANTLY IN THEIR ATOMIC FORM.RESONANCE LINES REPORTED CORRESPOND TO DOMINANT TRANSITIONS IN THE REGION BETWEen 300 AND 800 NM. For Fe SEe Fig. 10. For DOUBLET LINES WE GIVE THE MEAN WAVELENGTH.

\begin{tabular}{ccccrrc}
\hline Z & Element & $\begin{array}{c}\text { Atomic } \\
\text { Mass }\end{array}$ & $\begin{array}{c}\text { Mixing } \\
\text { Ratio }\end{array}$ & \multicolumn{2}{c}{$\begin{array}{c}\text { Ion. Limit } \\
\mathrm{eV}\end{array}$} & \multicolumn{1}{c}{$\lambda_{\text {Res }}$} \\
$\mathrm{nm}$
\end{tabular}

elements have large ionization potentials and they do not significantly affect the ionization of the alkali atoms deeper in the atmosphere. In order to demonstrate the dominance of the alkali atoms in the ionization of the lower atmosphere, here we only discuss the effects of their photoionization and electron recombination. Additional processes that could affect the profiles of the alkali atoms are discussed in section 4 .

Potassium has the most extended photoionization cross section reaching to $\sim 2900 \AA$. Photons at these wavelengths are not substantially absorbed by the other atmospheric gaseous compounds resulting in ionization of $\mathrm{K}$ to pressures greater than 1 bar (Fig. 4). For Na, photons available for ionization with $\lambda \leq 2412 \AA$ are also absorbed by other gaseous species, such as $\mathrm{H}_{2} \mathrm{O}$ and $\mathrm{H}_{2} \mathrm{~S}$ (as identified also by Fortney et al. (2003)) and photoionization is efficient down to $\sim 10^{-2}$ bar. At low pressures $\mathrm{Na}^{+}$and $\mathrm{K}^{+}$recombine radiatively with electrons. This process is slow, resulting in large electron densities. At deeper pressures, greater than $10^{-3} \mathrm{bar}, \mathrm{Na}^{+}$and $\mathrm{K}^{+}$are dominantly lost through 3 body recombination, which is much more rapid. Hence, most of the ionized alkali atoms return to their neutral state and the electron densities decrease (Fig. 5). At even higher pressures, larger than 0.1 bar, thermal ionization becomes efficient and the electron densities start to increase again. The 3-body recombination also becomes more efficient with increased pressure though, forcing the majority of the alkali atoms to remain in their neutral state.

The variable ionization regions of these atomic elements have their signature on the resulting electron densities (Fig. 6). For pressures lower than $10^{-8}$ bar electrons are produced from the ionization of atomic hydrogen, with secondary contributions by He and $\mathrm{C}$ photoionization. At higher pressures the majority of the electrons is produced by the photo-ionization of $\mathrm{Na}$ and $\mathrm{K}$, with the former dominating over the latter up to pressures of $\sim 10^{-2}$ bar. Below the 1 bar level photo-ionization rates are small and most of the electrons are produced by thermal ionization of K. Although thermal ionization produces large electron densities below the 1 bar level, it is important to note that photo-ionization dominates at lower pressures. The evaluation of the thermal electron 

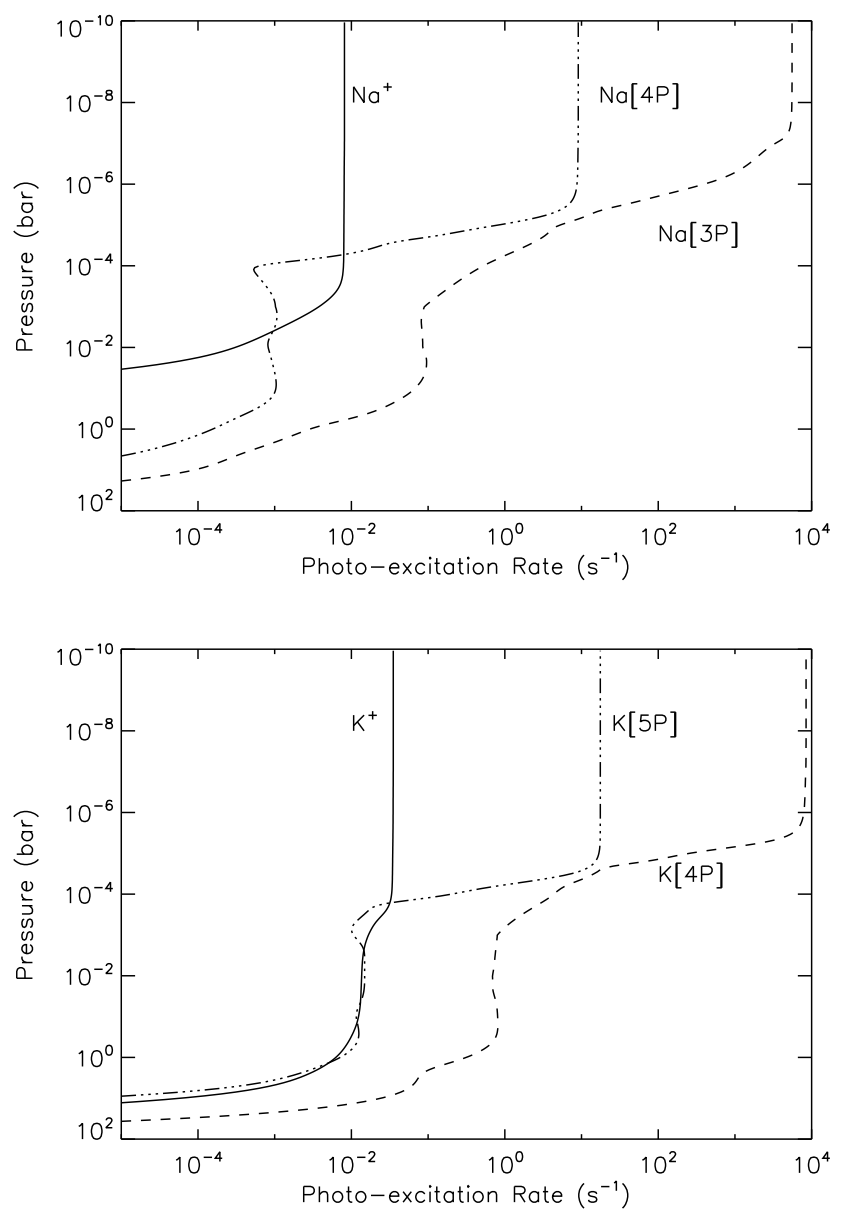

Fig. 4.- Photoionization rates (solid lines) of $\mathrm{Na}$ (top) and $\mathrm{K}$ (bottom). For comparison the corresponding rates for photoexcitation of different resonant states of are shown.

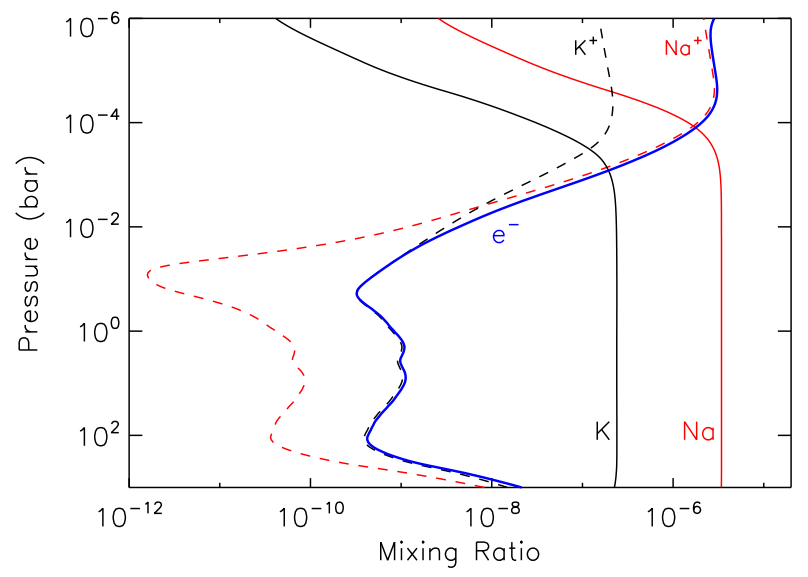

Fig. 5.- Vertical profiles for the mixing ratios of $\mathrm{Na}$ (red) and K (black) from our calculations. Solid lines show the neutral compound and dashed lines the corresponding ion. The total electron mixing ratio profile is presented by the blue line.

population based on the Saha equation considering all atomic elements used in the kinetic model shows that the thermal electron density at $10^{-2}$ bar should be $\sim 4 \times 10^{6}$ $\mathrm{cm}^{-3}$ (green line in Fig. 6). The photon-induced elec-

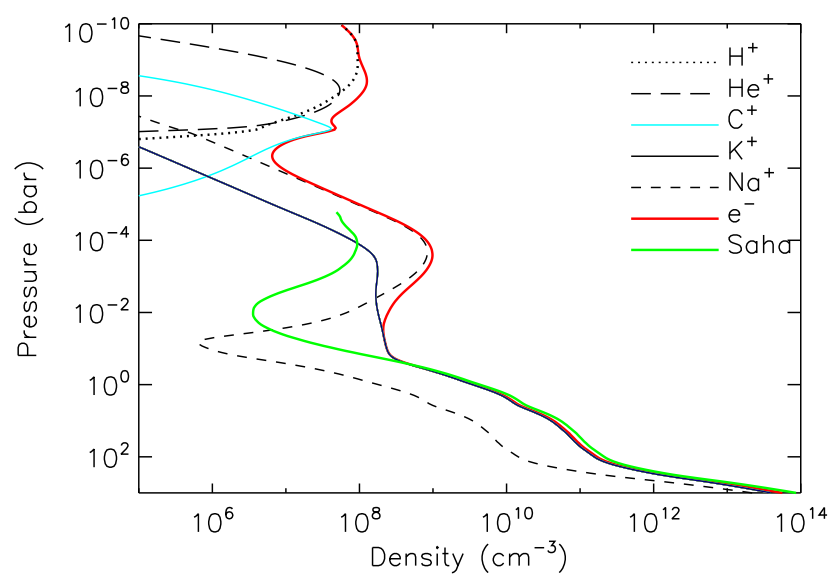

FIG. 6.- Calculated densities of different ions and the resulting electron density (solid red line) assuming charge balance, in the atmosphere of HD 209458 b. The green line presents the electron density assuming thermal ionization only.

tron density at the same level is almost 100 times larger. Such high electron densities so deep in the atmosphere are not common among the giant planets of our solar system, and for HD 209458 b result from the combination of strong stellar fluxes for this close-in exoplanet, and high atmospheric temperatures that allow $\mathrm{Na}$ and $\mathrm{K}$ to remain in atomic form. Thus, we can conclude that photo-ionization in the atmospheres of exoplanets similar to HD 209458 b will produce large electron densities in the middle atmosphere between $10 \mu$ bar and 1 bar.

\subsection{Transit depths}

We now turn our attention to the comparison of our model results with the available observations of alkali metals in HD 209458 b. Our opacities for the transit depth calculation include $\mathrm{H}_{2}$ Rayleigh scattering, absorption by $\mathrm{Na}$ and $\mathrm{K}$, collision induced absorption by $\mathrm{H}_{2}-\mathrm{H}_{2}$ and $\mathrm{H}_{2}$-He collisions, and molecular absorption by $\mathrm{H}_{2} \mathrm{O}$ (Fig. 7). We use the atomic line properties from the NIST database and we calculate the line profiles for the different pressure-temperature conditions of the model atmosphere assuming Voigt line profiles. For the alkali atoms we use the line parameters and broadening coefficients from Iro et al. (2005). We are aware of the divergence of the alkali line wings from the Voigt description at the far wings (Allard et al. 2003), but the conclusions we draw below are not affected by this issue. For the temperature profile we assume the average terminator profile (see Fig. 2), while for the chemical abundances we use the densities derived by the day side conditions since we expect that the strong zonal transport suggested by the GCMs will efficiently redistribute the abundances from the disk to the terminator (Showman et al. 2009). We discuss further below possible implications for the differences between the dawn and dusk terminator.

The transit depth at the sodium line appears to be consistent with the sodium wings observed by Sing et al. $(2008 \mathrm{~b})$ in the medium resolution STIS observations (Fig. [7). The analysis of the same observations by Knutson et al. (2007) and Deming et al. (2013) at larger spectral bins suggests the presence of a less broad $\mathrm{Na}$ wing, but our model is consistent with these observa- 

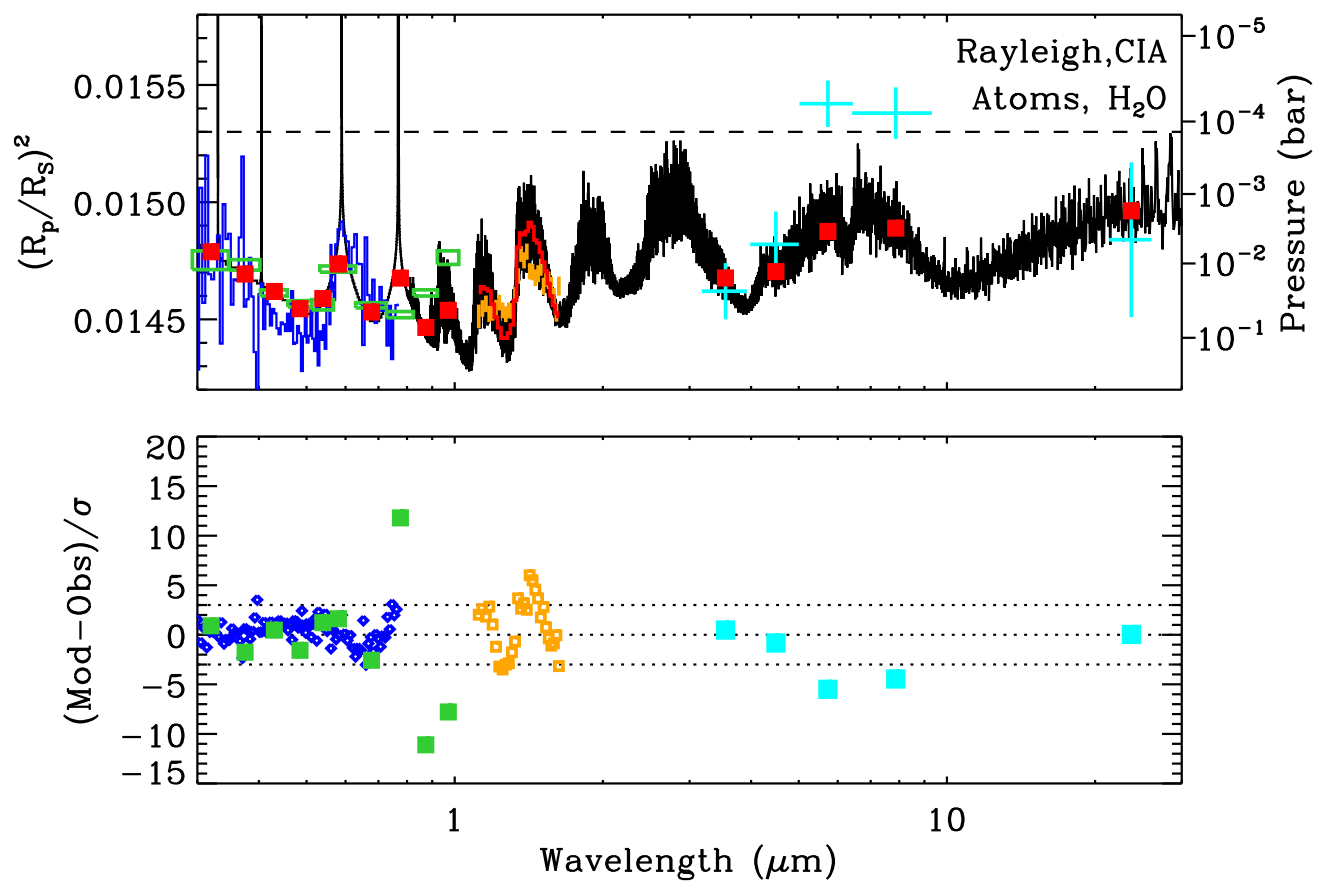

FIG. 7. - Transit depth calculation in the atmosphere of HD 209458 b. In the top panel, the black line is the transit depth based on our calculations including all atoms considered, Rayleigh scattering by $\mathrm{H}_{2}$, collision induced absorption, and $\mathrm{H}_{2} \mathrm{O}$. The green boxes present the results from Deming et al. (2013) from the re-analysis of the HST/STIS observations of Knutson et al. (2007), while the blue histogram presents the HST/STIS observations from Sing et al. (2008b). The orange lines present the HST/WFC observations from Deming et al. (2013). The cyan cross are the Spitzer transit depth measurements from IRAC (Beaulieu et al. 2010) and MIPS (Crossfield et al. 2012). The red points and line present our calculations mapped to the resolution of the observations. The dashed line presents the required cloud transit depth required to match the differential transit observations. In the lower panel we present the residuals between model and observations in terms of the 1 sigma level of uncertainty in the observations. The blue points correspond to the comparison with the Sing et al. (2008b) data, the green and orange squares to the Deming et al. (2013) data from STIS and WFC data, respectively, and the cyan squares to the Spitzer observations.

tions as well, given the larger uncertainty in the midresolution observations. The presence though or not of a broad wing at the sodium line has important implications for the interpretation of the observations and specifically the question of whether the atmosphere is clear or not. A broad atomic line wing implies a clear atmosphere, while a shallow atomic core would indicate the presence of clouds (or haze) at the probed altitudes.

A closer look at the $\mathrm{Na}$ line double core (Fig. 8) shows that our model generates a differential transit depth that is larger than the original observations by Charbonneau et al. (2002) and the subsequent observations by Sing et al. (2008b). The very low differential transit depth at the sodium line core was originally interpreted as an indication of a cloud deck at the probed pressure levels around the sodium core that reduced the contrast between the line center and the wings (Charbonneau et al. 2002). We find that such an interpretation is not consistent with the subsequent observations of Rayleigh scattering at shorter wavelengths, and in general with the low resolution spectra. This argument becomes obvious if we assume a spectrally flat cloud deck around the $\mathrm{Na}$ core that would be consistent with the observed differential transit depth values. Our calculations suggest that this would occur for a cloud transit depth close to 0.00153 (see Fig. 8), which results in a differential transit depth that is consistent with both the magnitude and the spectral dependence of the observations. However, such a cloud would be inconsistent with the observed transit depth at shorter wavelengths since it has a larger absorption than the strongest absorption observed at $0.3 \mu \mathrm{m}$ (see dashed line in Fig. (7). In other words the cloud properties implied by the high resolution differential transit depth are not consistent with the $\mathrm{Na}$ wing detection and the observed slope at shorter wavelength $\$ 3$. We further discuss the implications of this result below.

At longer wavelengths, absorption by $\mathrm{H}_{2} \mathrm{O}$ alone provides a consistent picture with the HST observations by Deming et al. (2013) between 1.1 and 1.6 microns, and the Spitzer observations at longer wavelengths by Beaulieu et al. (2010) and Crossfield et al. (2012), although the model seems to lack some opacity at 5.8 and 8 microns.

The most striking feature of the comparison of the model with the observations is the large transit depth at the potassium line in the model relative to practically no detection in the observations (Knutson et al. 2007; Sing et al. 2008b; Jensen et al. 2011). It is commonly anticipated that the lack of potassium could be explained

${ }^{3}$ In reality the cloud spectral signature will not be flat over the whole spectral range of the observations, but for small particles (as expected at the pressure regions of interest here) the absorption would increase towards shorter wavelengths and decrease towards longer wavelengths around the sodium core. Such a spectral behavior would make the cloud scenario even less consistent with the differential transit depth observations, relative to the spectrally flat case. 

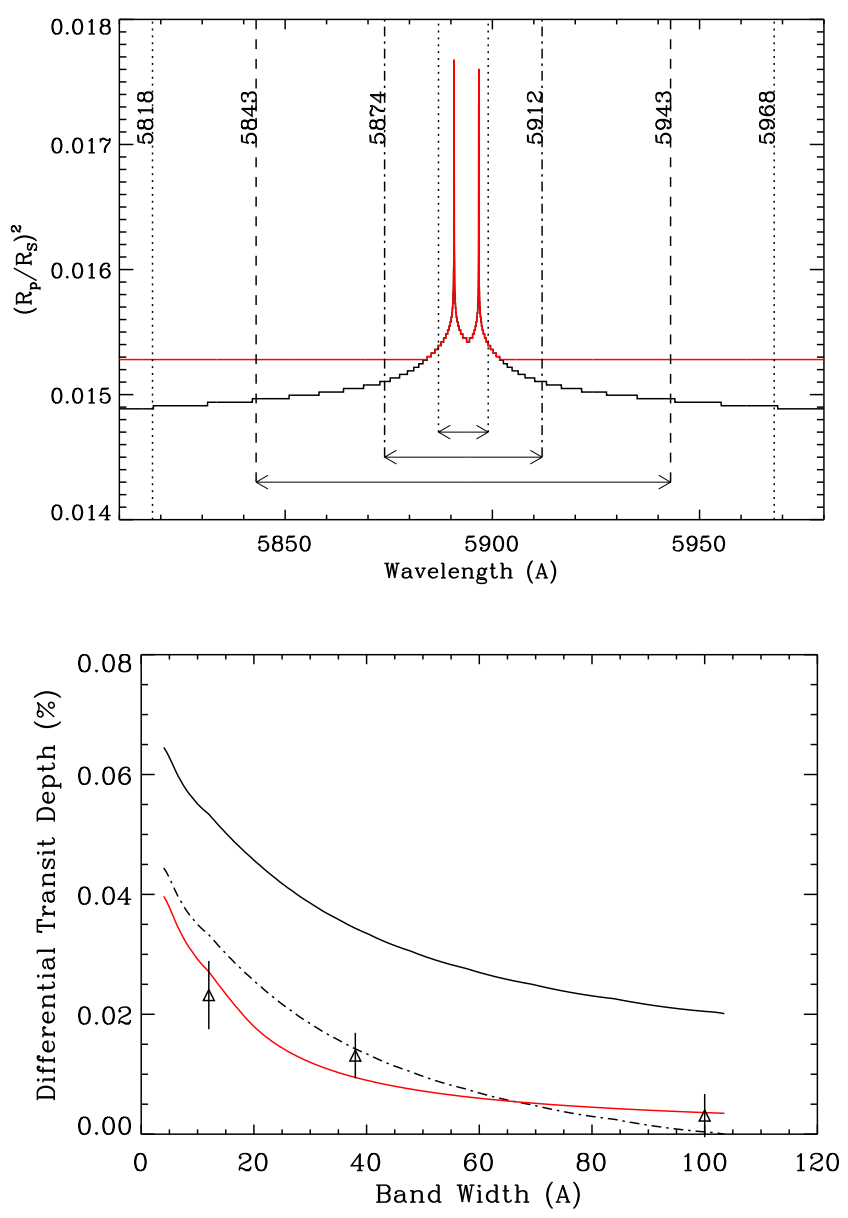

FIG. 8.- Top: Transit depth spectrum around the Na line core. The black line corresponds to the clear atmosphere case and the red line to the case with a cloud deck. The vertical broken lines designate the bands used by Charbonneau et al. (2002) for the investigation of the differential transit depth assuming band widths of 12,38 , and $100 \AA$ (arrows). Bottom: Differential transit depth calculation performed by assuming different band widths around the $\mathrm{Na}$ core and comparing with the reference region bound between 5818 and $5968 \AA$. The solid line is the clear atmosphere case assuming the $\mathrm{Na}$ profile from our photochemical model, while the dash-dotted line corresponds to the clear atmosphere case but homogeneously shifted downwards by $\sim 0.02 \%$. The red line corresponds to the differential transit depth assuming a cloud deck with a transit depth of $\sim 0.0153$ around the sodium core. The symbols with the associated error bars are the differential transit depth values from the Charbonneau et al. (2002) HST/STIS observations.

by the rapid photo-ionization of this element due to its very low ionization potential. Our transit depth calculations imply that for the assumed solar elemental abundances, the K profile would have to be depleted to very deep levels in the atmosphere (close to 10 mbar) in order to be consistent with the observations. Our photochemical calculations presented above demonstrate that this is not likely. Before considering other options to explain this discrepancy between observations and modeling, we decided to further investigate the alkali chemistry in order to verify that other photochemical processes can not help reduce the abundances of $\mathrm{Na}$ and $\mathrm{K}$. These studies are described in section 4 .

\subsection{Other atoms}

We have also considered the possibility of other atoms in the atmospheres of hot extrasolar giant planets. For example, $\mathrm{Mg}, \mathrm{Si}, \mathrm{Ca}, \mathrm{Al}$, and $\mathrm{Fe}$ combine a low ionization potential with a high elemental abundance relative to other heavy atoms (Table 11) and could contribute to the electron densities. Most of these heavy atoms should condense in the low atmosphere for the temperature conditions assumed (see Fig. 2), in which case they would not significantly contribute to the ionosphere. The observations of HD $209458 \mathrm{~b}$ seem to favor an atmosphere in which $\mathrm{Si}$, at least, survives in the upper atmosphere. Specifically, the signature of SiIII in the transit depth observations (Linsky et al. 2010), implies a large abundance of atomic silicon in the thermosphere, which should not be possible if condensates such as forsterite or enstatite form in the lower atmosphere (Koskinen et al. 2013a, b). Based on these observations and interpretation, we performed calculations including the above atoms in order to evaluate their potential impact on the electron densities and the transit depth.

We used ionization cross sections for all new atoms from Verner et al. (1996). We could not find recombination rates for all elements considered, thus certain estimations had to be made based on the electronic structure of each element. For $\mathrm{Mg}$ and $\mathrm{Si}$ we used recombination rates from Aldrovandi and Pequignot (1973) and for $\mathrm{Ca}$ we assumed the same rate as for $\mathrm{Mg}$. For $\mathrm{Al}$ we estimated the recombination rate with that of SiIII from Aldrovandi and Pequignot (1973), while for Fe we used the recombination rate from Nahar et al. (1997). Three body rates were estimated based on the rate for sodium. Based on the NASA thermochemistry model (Burcat and Ruscid 2005), for the atmospheric conditions of HD209458b, the most abundant form of the heavy elements is the atomic, with the exception of $\mathrm{Si}$ and Al. Aluminum at the lower boundary is dominantly in the form of $\mathrm{AlOH}$ and $\mathrm{AlH}$, which we follow kinetically in the model through the reactions:

$$
\begin{gathered}
\mathrm{Al}+\mathrm{H}_{2} \mathrm{O} \leftrightarrow \mathrm{AlOH}+\mathrm{H} \\
\mathrm{Al}+\mathrm{H}+\mathrm{M} \leftrightarrow \mathrm{AlH}+\mathrm{M}
\end{gathered}
$$

with rates for the forward reactions taken from McClean et al. (1993); Sharipov et al. (2011), and Swihart et al. (2003), respectively. Silicon, like aluminum, is present in molecular compounds at the lower boundary with $\mathrm{SiO}$ the most abundant species. For this case though there is a large number of other silicon species that can be formed. In our calculations we have followed kinetically the transition between $\mathrm{SiO}$ and $\mathrm{SiH}_{4}$ through different intermediate species, as well as the interaction of silicon with other elements to form $\mathrm{SiS}, \mathrm{SiN}$, and $\mathrm{SiC}$. The $\mathrm{SiO}-\mathrm{SiH}_{4}$ equilibrium is described kinetically with a scheme similar to the $\mathrm{CO}-\mathrm{CH}_{4}$ equilibrium (Moses et al. 2011), but modified by the thermochemical parameters of the silicon-based species. For the most part of the atmosphere $\mathrm{SiO}$ and $\mathrm{SiH}_{4}$ remain close to their equilibrium abundances. The details of these calculations are beyond the scope of the current study and will be presented in a future study.

According to our calculations, the two aluminum molecular compounds have similar abundances at the lower boundary but diverge at lower pressures with $\mathrm{AlOH}$ becoming the most abundant species close to $10^{2}$ bar (Fig. 9). At pressures below $10^{-2}$ bar, reaction 

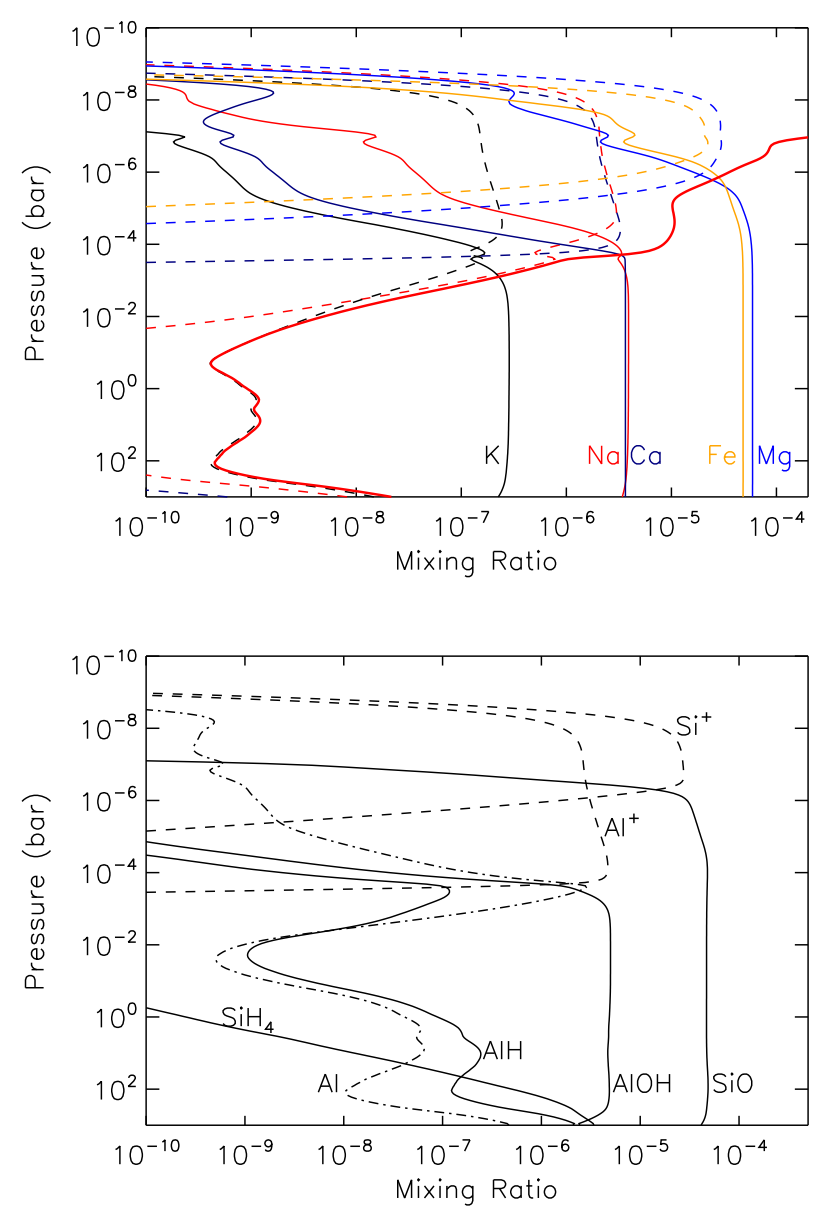

FIG. 9.- Vertical profiles for the mixing ratios of atoms and their corresponding ions included in the calculations. The top panel presents the profiles for elements that are present in their atomic form and bottom panel presents the case of aluminum and silicon species that are present in molecular form at the lower boundary. Solid lines show the neutral compound and dashed lines the corresponding ion. For clarity the Al profile is presented with a dash-dotted line.

with atomic hydrogen gradually returns aluminum to its atomic form before becoming mainly ionized at pressures lower than $10^{-3}$ bar. Calcium has a similar ionization pressure region since its ionization limit is close to that of aluminum. The remaining elements $\mathrm{Mg}, \mathrm{Fe}$ and $\mathrm{Si}$ have all significantly higher ionization potentials compared to the previous elements, and their corresponding ions become important only above $10^{-6}$ bar. $\mathrm{Mg}, \mathrm{Fe}$, and $\mathrm{Si}$ photo-ionization contributes to the local increase of the electron density close to $10^{-6}$ bar, while the signature of $\mathrm{Ca}$ and $\mathrm{Al}$ photo-ionization is clear close to $10^{-4}$ bar. The increased electron abundance also affects the $\mathrm{Na}$ and $\mathrm{K}$ profiles close to $10^{-4}$ due to the enhanced recombination rates.

Inclusion of these new atomic profiles to the transit depth calculations produces numerous narrow absorption lines at short wavelengths (see also Barman 2007). Although most of these lines are too narrow to be detectable with the current instrumentation, some of them, originating mainly from $\mathrm{Ca}(\sim 423 \mathrm{~nm})$ and $\mathrm{Fe}$ (all other broad absorption features between 0.3 and $0.4 \mu \mathrm{m}$ ), are wide enough to be observed. The medium resolution
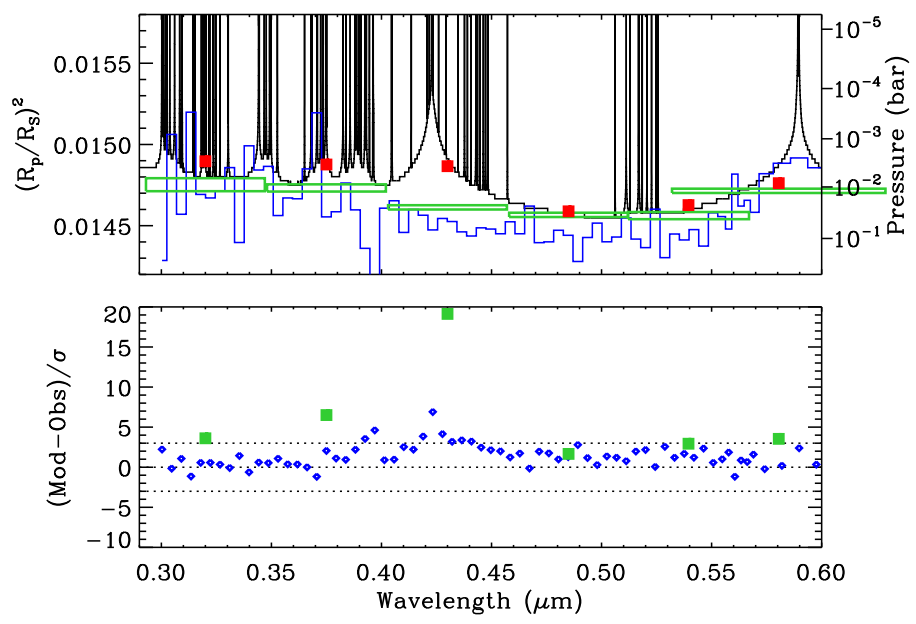

FIG. 10. - Same as Fig. 7 but with the inclusion of the extra atomic abundances calculated by the model.

HST/STIS observations (Sing et al. 2008b) demonstrate some structure in this part of the spectrum, but they suffer from large uncertainties that do not allow for a clear assignment of absorption signatures. On the other hand, the low resolution analysis of the same observations (Knutson et al. 2007; Deming et al. 2013) do not indicate the presence of such absorption features, which would affect the observed transit depth according to our calculations (Fig. 10). The residuals between the model and the broadband observations are about 6 and 20 sigma at the major $\mathrm{Fe}$ and $\mathrm{Ca}$ absorption signatures, respectively, while close to the calcium line the residuals relative to the medium resolution observations are more than three sigma. These results indicate that the abundances of $\mathrm{Ca}$ and $\mathrm{Fe}$, at least, are decreased at the probed pressure levels of the HD $209458 \mathrm{~b}$ relative to the solar elemental abundances, a behavior anticipated due to condensation. Therefore we decided to consider atomic opacities of $\mathrm{Na}$ and $\mathrm{K}$ only for the remainder of this study.

\section{EXTENDED ALKALI CHEMISTRY}

The extended processes of alkali chemistry we consider in the model include the simulation of molecular chemistry, as well as of excited atomic state processes that could potentially reduce the abundances of $\mathrm{Na}$ and $\mathrm{K}$. In addition, we investigate more aspects of their ion chemistry. An overview of the reactions involved and the rates used is provided in Tables 2 \& 3

\subsection{Molecular states}

For the temperatures considered here, alkali atoms can form molecular structures. We follow the populations of $\mathrm{NaH}, \mathrm{NaOH}, \mathrm{KOH}$, and $\mathrm{KH}$, which are the thermochemically most abundant molecular structures at the thermal conditions assumed. We follow these populations kinetically so that we can identify any potential increases of their densities above the (otherwise low) thermochemical levels, due to quenching by photochemistry and dynamics. The chemical processes we consider for the description of these species are similar for $\mathrm{Na}$ and $\mathrm{K}$ (although with different rates for each element). For example, reaction of $\mathrm{Na}$ with water leads to $\mathrm{NaOH}$ : 

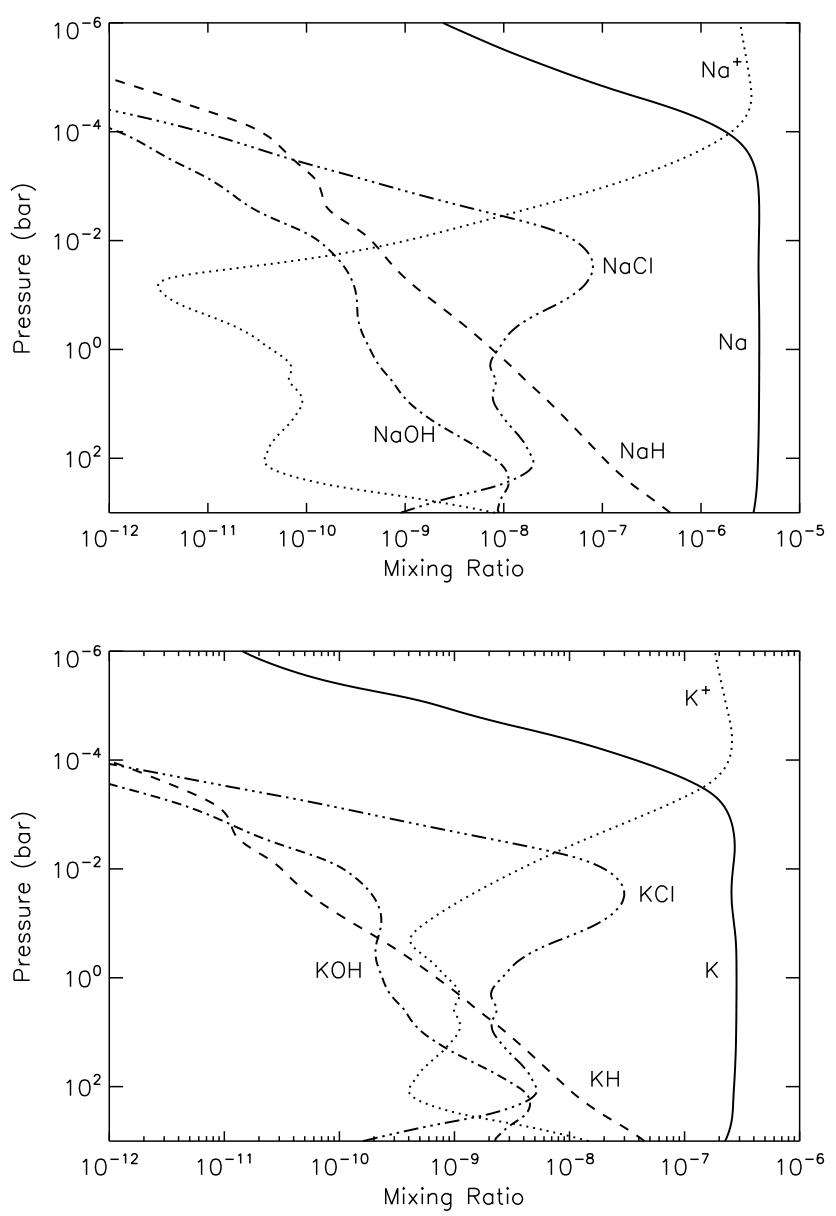

FIG. 11.- Model results for the mixing ratios of different molecular structures of sodium (top) and potassium (bottom).

$$
\mathrm{Na}+\mathrm{H}_{2} \mathrm{O} \leftrightarrow \mathrm{NaOH}+\mathrm{H},
$$

while reaction with hydrogen forms $\mathrm{NaH}$ :

$$
\mathrm{Na}+\mathrm{H}_{2} \leftrightarrow \mathrm{NaH}+\mathrm{H}
$$

These molecules can thermally decompose back to sodium and the corresponding radicals:

$$
\begin{gathered}
\mathrm{NaOH}+\mathrm{M} \leftrightarrow \mathrm{Na}+\mathrm{OH}+\mathrm{M} \\
\mathrm{NaH}+\mathrm{M} \leftrightarrow \mathrm{Na}+\mathrm{H}+\mathrm{M},
\end{gathered}
$$

with both forward and reverse pathways important depending on the assumed temperature conditions. For the cases studied here the formation of these molecules is favored only deep in the atmosphere and their resulting abundance is always smaller than the atomic (Fig. 11).

We also track the populations of $\mathrm{NaCl}$ and $\mathrm{KCl}$, which require the inclusion of $\mathrm{Cl}$ and $\mathrm{HCl}$ in the study. Deep in the atmosphere chlorine is dominantly in the form of $\mathrm{HCl}$ in a balance with atomic $\mathrm{Cl}$ that is controlled through the reaction:

$$
\mathrm{HCl}+\mathrm{H} \leftrightarrow \mathrm{Cl}+\mathrm{H}_{2},
$$

while at lower pressures reaction with alkali atoms leads to

$$
\mathrm{Na}, \mathrm{K}+\mathrm{HCl} \leftrightarrow \mathrm{NaCl}, \mathrm{KCl}+\mathrm{H}
$$

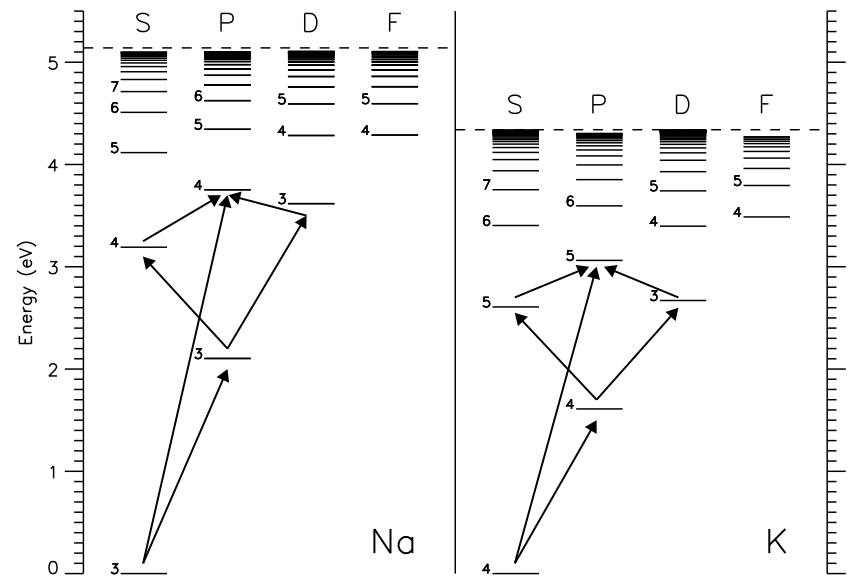

FIG. 12.- Energy level diagram of the electronic states of $\mathrm{Na}$ and K. Arrows indicate the photon excitations considered in the calculations for the first four excited states of each atom. Dashed lines present the ionization limits.

that affects the $\mathrm{HCl}$ population to a small degree. We also consider the reaction

$$
\mathrm{HCl}+\mathrm{OH} \leftrightarrow \mathrm{H}_{2} \mathrm{O}+\mathrm{Cl},
$$

but found that it has a third role in the overall chlorine species profiles, after alkali atoms. Photolysis of $\mathrm{HCl}$ has a minor impact on its abundance and we included it with cross sections from Brion et al. (2005). The results show that close to the temperature minimum the abundances of both $\mathrm{NaCl}$ and $\mathrm{KCl}$ increase. $\mathrm{KCl}$ becomes large enough close to $10 \mathrm{mbar}$ that induces a small decrease in the atomic potassium abundance (Fig. 11). This decrease though is too small to reduce the transit depth of the potassium line to the level of the observations. Hence, molecular chemistry can not explain the low alkali abundances. Another conclusion we can derive from these results is that, notwithstanding the small differences in the profiles of the different species involved, $\mathrm{Na}$ and $\mathrm{K}$ have a very similar chemical behavior, as anticipated.

\subsection{Excited states}

Due to the large photon fluxes at visible wavelengths and the large oscillator strengths for the alkali resonant transitions, the photo-excitation rates of these states are orders of magnitude larger than the corresponding photoionization rates (Fig. 4). Therefore a large population of excited alkali atoms can potentially exist in the exoplanet atmospheres. Processes forbidden or weak for ground state atoms could be efficient for the excited states and in this way drive the system further away from the thermochemical equilibrium. Moreover, if the resulting atomic state population of $\mathrm{Na}$ and $\mathrm{K}$ is much different from the LTE abundances, further modifications in the transit depth would be expected (Barman et al. 2002). Thus, a detailed description of these processes is necessary in order to evaluate their role in the abundances of alkali atoms.

Two electronic states of $\mathrm{Na}$ and $\mathrm{K}$ can be efficiently excited from their grounds states directly. These are $3\left({ }^{2} \mathrm{P}\right)$ and $4\left({ }^{2} \mathrm{P}\right)$ for $\mathrm{Na}$ and $4\left({ }^{2} \mathrm{P}\right)$ and $5\left({ }^{2} \mathrm{P}\right)$ for $\mathrm{K}$ (Fig. 12). For simplicity we reduce the nomenclature of these states 
TABLE 2

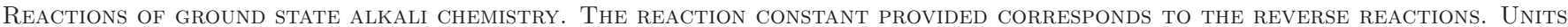
ARE IN CGS.

\begin{tabular}{|c|c|c|c|c|}
\hline & Reaction & Forward Rate & Reaction Constant & Notes/Reference \\
\hline $\mathrm{R} 1$ & $\mathrm{Na}+h \nu \rightarrow \mathrm{Na}^{+}+\mathrm{e}^{-}$ & & & \\
\hline $\mathrm{R} 2$ & $\mathrm{Na}^{+}+\mathrm{e}^{-} \rightarrow \mathrm{Na}+h \nu$ & $\mathrm{k}=1 . \times 10^{-11} \exp (-50895 / T)$ & & Verner et al. (1996) \\
\hline $\mathrm{R} 4$ & $\mathrm{Na}^{+}+\mathrm{e}^{-}+\mathrm{M} \rightleftharpoons \mathrm{Na}+\mathrm{M}$ & $\begin{array}{l}\mathrm{k}_{0}=3.43 \times 10^{-14} \mathrm{~T}^{-3.77} \\
\mathrm{k}_{\infty}=1 . \times 10^{-7}\end{array}$ & $2.288 \times 10^{+15} \mathrm{~T}^{1.506} \exp (-59630 / \mathrm{T})$ & $\frac{\text { Su et al. }(2001)}{\text { Est }}$ \\
\hline R6 & $\mathrm{NaH}+\mathrm{H} \rightleftharpoons \mathrm{Na}+\mathrm{H}_{2}$ & $\mathrm{k}=2.38 \times 10^{-12} \mathrm{~T}^{0.69} \exp (-2360 / T)$ & $1.160 \times 10^{-01} \mathrm{~T}^{0.636} \exp (-29990 / \mathrm{T})$ & Mayer et al. (1966) \\
\hline $\mathrm{R} 8$ & $\mathrm{Na}+\mathrm{H}_{2} \mathrm{O} \rightleftharpoons \mathrm{NaOH}+\mathrm{H}$ & $\mathrm{k}=4.07 \times 10^{-10} \exp (-21900 / T)$ & $3 \times 10^{3} \mathrm{~T}^{-1.293} \exp (19210 / \mathrm{T})$ & Jensen (1982) \\
\hline R10 & $\mathrm{OH}+\mathrm{Na}+\mathrm{M} \rightleftharpoons \mathrm{NaOH}+\mathrm{M}$ & $\begin{array}{l}\mathrm{k}_{0}=1.9 \times 10^{-25} \mathrm{~T}^{-2.21} \exp (-41 / \mathrm{T}) \\
\mathrm{k}_{\infty}=1 . \times 10^{-11}\end{array}$ & $4.423 \times 10^{+19} \mathrm{~T}^{1.115} \exp (-38360 / \mathrm{T})$ & Patrick and Golden (1984) \\
\hline $\mathrm{R} 12$ & $\mathrm{H}+\mathrm{Na}+\mathrm{M} \rightleftharpoons \mathrm{NaH}+\mathrm{M}$ & $\begin{array}{l}\mathrm{k}_{0}=1.9 \times 10^{-25} \mathrm{~T}^{-2.21} \exp (-41 / \mathrm{T}) \\
\mathrm{k}_{\infty}=1 . \times 10^{-11}\end{array}$ & $4.873 \times 10^{+19} \mathrm{~T}^{0.912} \exp (-20940 / \mathrm{T})$ & Est as $\mathrm{OH}+\mathrm{Na}+\mathrm{M}$ \\
\hline R14 & $\mathrm{Na}+\mathrm{H} \rightleftharpoons \mathrm{Na}^{+}+\mathrm{H}^{-}$ & $\mathrm{k}=1 . \times 10^{-11} \exp (-50895 / T)$ & $2.81 \times 10^{20} \mathrm{~T}^{-5.365} \exp (47650 / \mathrm{T})$ & Est \\
\hline R16 & $\mathrm{Na}^{+}+\mathrm{K} \rightleftharpoons \mathrm{K}^{+}+\mathrm{Na}$ & $\mathrm{k}=1 . \times 10^{-11}$ & $7.844 \times 10^{-01} \mathrm{~T}^{0.032} \exp (-9244 / \mathrm{T})$ & Est \\
\hline R17 & $\mathrm{K}+h \nu \rightarrow \mathrm{K}^{+}+\mathrm{e}^{-}$ & & & \\
\hline R18 & $\mathrm{K}^{+}+\mathrm{e}^{-} \rightarrow \mathrm{K}+h \nu$ & $\mathrm{k}_{0}=2.66 \times 10^{-25} \mathrm{~T}^{-2.21} \exp (-48 / \mathrm{T})$ & & Verner et al. (1996) \\
\hline R20 & $\mathrm{K}^{+}+\mathrm{e}^{-}+\mathrm{M} \rightleftharpoons \mathrm{K}+\mathrm{M}$ & $\begin{array}{l}\mathrm{k}_{0}=3.43 \times 10^{-14} \mathrm{~T}^{-3.77} \\
\mathrm{k}_{\infty}=1 . \times 10^{-7}\end{array}$ & $3.885 \times 10^{+16} \mathrm{~T}^{1.155} \exp (-50680 / \mathrm{T})$ & Est as $\mathrm{Na}^{+}+\mathrm{e}^{-}$ \\
\hline R22 & $\mathrm{KH}+\mathrm{H} \rightleftharpoons \mathrm{K}+\mathrm{H} 2$ & $\mathrm{k}=2.38 \times 10^{-12} \mathrm{~T}^{0.69} \exp (-2360 / T)$ & $1.326 \times 10^{-01} \mathrm{~T}^{0.678} \exp (-30370 / \mathrm{T})$ & Est as $\mathrm{NaH}$ \\
\hline $\mathrm{R} 24$ & $\mathrm{~K}+\mathrm{H}_{2} \mathrm{O} \rightleftharpoons \mathrm{KOH}+\mathrm{H}$ & $\mathrm{k}=5 \cdot \times 10^{-10} \exp (-20000 / T)$ & $1.789 \times 10^{+03} \mathrm{~T}^{-1.277} \exp (16550 / \mathrm{T})$ & Jensen et al. (1979) \\
\hline R26 & $\mathrm{OH}+\mathrm{K}+\mathrm{M} \rightleftharpoons \mathrm{KOH}+\mathrm{M}$ & $\begin{array}{l}\mathrm{k}_{0}=2.66 \times 10^{-25} \mathrm{~T}^{-2.21} \exp (-48 / \mathrm{T}) \\
\mathrm{k}_{\infty}=1 . \times 10^{-11}\end{array}$ & $8.414 \times 10^{+18} \mathrm{~T}^{1.265} \exp (-40910 / \mathrm{T})$ & $\frac{\text { Patrick and Golden (1984) }}{\text { Est }}$ \\
\hline R28 & $\mathrm{H}+\mathrm{K}+\mathrm{M} \rightleftharpoons \mathrm{KH}+\mathrm{M}$ & $\begin{array}{l}\mathrm{k}_{0}=2.66 \times 10^{-25} \mathrm{~T}^{-2.21} \exp (-48 / \mathrm{T}) \\
\mathrm{k}_{\infty}=1 . \times 10^{-11}\end{array}$ & $4.423 \times 10^{+19} \mathrm{~T}^{0.866} \exp (-20570 / \mathrm{T})$ & Est as $\mathrm{OH}+\mathrm{K}+\mathrm{M}$ \\
\hline R30 & $\mathrm{HCl}+h \nu \rightarrow \mathrm{H}+\mathrm{Cl}$ & & & \\
\hline R32 & $\mathrm{H}+\mathrm{HCl} \rightleftharpoons \mathrm{H}_{2}+\mathrm{Cl}$ & $\mathrm{k}=2.4 \times 10^{-11} \exp (-1730 / T)$ & $1.795 \mathrm{~T}^{-0.005} \exp (-478 / \mathrm{T})$ & Allison et al. (1996) \\
\hline R34 & $\mathrm{HCl}+\mathrm{OH} \rightleftharpoons \mathrm{H}_{2} \mathrm{O}+\mathrm{Cl}$ & $\mathrm{k}=6.84 \times 10^{-19} \mathrm{~T}^{2.12} \exp (646 / T)$ & $3.301 \times 10^{+01} \mathrm{~T}^{-0.183} \exp (-8030 / \mathrm{T})$ & Bryukov et al. (2006) \\
\hline R36 & $\mathrm{K}+\mathrm{HCl} \rightleftharpoons \mathrm{KCl}+\mathrm{H}$ & $\mathrm{k}=5.6 \times 10^{-10} \exp (-4170 / T)$ & $2.707 \times 10^{+01} \mathrm{~T}^{-0.648} \exp (710 / \mathrm{T})$ & Husain and Lee (1988) \\
\hline R38 & $\mathrm{Na}+\mathrm{HCl} \rightleftharpoons \mathrm{NaCl}+\mathrm{H}$ & $\mathrm{k}=4 . \times 10^{-10} \exp (-4090 / T)$ & $3.825 \times 10^{+01} \mathrm{~T}^{-0.646} \exp (2444 / \mathrm{T})$ & Husain and Marshall (1986) \\
\hline R40 & $\mathrm{HCl}+\mathrm{M} \rightleftharpoons \mathrm{H}+\mathrm{Cl}+\mathrm{M}$ & $\mathrm{k}_{0}=7.31 \times 10^{-11} \exp (-41000 / \mathrm{T})$ & $6.4543320 \times 10^{-19} \mathrm{~T}^{-1.632} \exp (50360 / \mathrm{T})$ & Baulch et al. (1981) \\
\hline
\end{tabular}

TABLE 3

Reactions of excited state alkali chemistry. See Table 2 for Details and text for discussion of the estimated rates.

\begin{tabular}{|c|c|c|c|c|}
\hline & Reaction & Forward Rate & Reaction Constant & Notes/Reference \\
\hline R41 & $\mathrm{Na}[3 \mathrm{P}] \rightleftharpoons \mathrm{Na}+h \nu$ & $\mathrm{k}=6.15 \times 10^{7}$ & & Kramida et al. (2013) \\
\hline R42 & $\mathrm{Na}[4 \mathrm{P}] \rightleftharpoons \mathrm{Na}+h \nu$ & $\mathrm{k}=2.74 \times 10^{6}$ & & Kramida et al. (2013) \\
\hline R43 & $\mathrm{Na}[3 \mathrm{D}] \rightleftharpoons \mathrm{Na}[3 \mathrm{P}]+h \nu$ & $\mathrm{k}=3.43 \times 10^{7}$ & & Kramida et al. (2013) \\
\hline R44 & $\mathrm{Na}[4 \mathrm{~S}] \rightleftharpoons \mathrm{Na}[3 \mathrm{P}]+h \nu$ & $\mathrm{k}=1.32 \times 10^{7}$ & & Kramida et al. (2013) \\
\hline R45 & $\mathrm{Na}[4 \mathrm{P}] \rightleftharpoons \mathrm{Na}[4 \mathrm{~S}]+h \nu$ & $\mathrm{k}=6.63 \times 10^{6}$ & & Kramida et al. (2013) \\
\hline R46 & $\mathrm{Na}[4 \mathrm{P}] \rightleftharpoons \mathrm{Na}[3 \mathrm{D}]+h \nu$ & $\mathrm{k}=1.05 \times 10^{5}$ & & Kramida et al. (2013) \\
\hline R48 & $\mathrm{Na}[3 \mathrm{P}]+\mathrm{M} \rightleftharpoons \mathrm{Na}+\mathrm{M}$ & $\mathrm{k}=1 . \times 10^{-11} \mathrm{~T}^{-0.50}$ & 1. $\exp (-24239 / \mathrm{T})$ & Earl and Herm (1974) \\
\hline R49 & $\mathrm{Na}[3 \mathrm{P}, 4 \mathrm{~S}, 3 \mathrm{D}, 4 \mathrm{P}]+h \nu \rightarrow \mathrm{Na}^{+}+\mathrm{e}^{-}$ & & & \\
\hline R51 & $\mathrm{Na}[3 \mathrm{P}]+\mathrm{H}_{2} \mathrm{O} \rightleftharpoons \mathrm{NaOH}+\mathrm{H}$ & $\mathrm{k}=2.2 \times 10^{-6} \exp (-21900 / T)$ & $3 \times 10^{3} \mathrm{~T}^{-1.293} \exp (-5119 / \mathrm{T})$ & Muller et al. (1980); Jensen (1982) \\
\hline R53 & $\mathrm{Na}[3 \mathrm{P}]+\mathrm{HCl} \rightleftharpoons \mathrm{NaCl}+\mathrm{H}$ & $\mathrm{k}=1.6 \times 10^{-12} \mathrm{~T}^{0.50}$ & $3.825 \times 10^{1} \mathrm{~T}^{-0.646} \exp (-21905 / \mathrm{T})$ & Weiss et al. (1988) \\
\hline R55 & $\mathrm{Na}[4 \mathrm{~S}]+\mathrm{M} \rightleftharpoons \mathrm{Na}+\mathrm{M}$ & $\mathrm{k}=9 . \times 10^{-12} \mathrm{~T}^{-0.50}$ & 1. $\exp (-36777 / \mathrm{T})$ & Est as $\mathrm{Na}[4 \mathrm{P}]$ \\
\hline R57 & $\mathrm{Na}[4 \mathrm{~S}]+\mathrm{H}_{2} \rightleftharpoons \mathrm{NaH}+\mathrm{H}$ & $\mathrm{k}=9 . \times 10^{-12} \mathrm{~T}^{-0.50}$ & $8.62 \mathrm{~T}^{-0.636} \exp (-6777 / \mathrm{T})$ & Est as $\mathrm{Na}[4 \mathrm{P}]$ \\
\hline R59 & $\mathrm{Na}[4 \mathrm{~S}]+\mathrm{H}_{2} \mathrm{O} \rightleftharpoons \mathrm{NaOH}+\mathrm{H}$ & $\mathrm{k}=2.2 \times 10^{-6} \exp (-21900 / T)$ & $3 \times 10^{3} \mathrm{~T}^{-1.293} \exp (-17657 / \mathrm{T})$ & Est as $\mathrm{Na}[3 \mathrm{P}]$ \\
\hline R61 & $\mathrm{Na}[3 \mathrm{D}]+\mathrm{M} \rightleftharpoons \mathrm{Na}+\mathrm{M}$ & $\mathrm{k}=9 . \times 10^{-12} \mathrm{~T}^{-0.50}$ & 1. $\exp (-41682 / \mathrm{T})$ & Est as $\mathrm{Na}[4 \mathrm{P}]$ \\
\hline R63 & $\mathrm{Na}[3 \mathrm{D}]+\mathrm{H}_{2} \rightleftharpoons \mathrm{NaH}+\mathrm{H}$ & $\mathrm{k}=9 . \times 10^{-12} \mathrm{~T}^{0.50}$ & $8.62 \mathrm{~T}^{-0.636} \exp (-11682 / \mathrm{T})$ & Est as $\mathrm{Na}[4 \mathrm{P}]$ \\
\hline R65 & $\mathrm{Na}[3 \mathrm{D}]+\mathrm{H}_{2} \mathrm{O} \rightleftharpoons \mathrm{NaOH}+\mathrm{H}$ & $\mathrm{k}=2.2 \times 10^{-6} \exp (-21900 / T)$ & $3 \times 10^{3} \mathrm{~T}^{-1.293} \exp (-22562 / \mathrm{T})$ & Est as $\mathrm{Na}[3 \mathrm{P}]$ \\
\hline R67 & $\mathrm{Na}[4 \mathrm{P}]+\mathrm{M} \rightleftharpoons \mathrm{Na}[3 \mathrm{D}]+\mathrm{M}$ & $\mathrm{k}=9 . \times 10^{-12} \mathrm{~T}-0.50$ & 1. $\exp (-1567 / \mathrm{T})$ & Kleiber et al. (1993) \\
\hline R69 & $\mathrm{Na}[4 \mathrm{P}]+\mathrm{H}_{2} \rightleftharpoons \mathrm{NaH}+\mathrm{H}$ & $\mathrm{k}=9 . \times 10^{-12} \mathrm{~T}^{0.50}$ & $8.62 \mathrm{~T}^{-0.636} \exp (-13249 / \mathrm{T})$ & Kleiber et al. (1993) \\
\hline R71 & $\mathrm{Na}[4 \mathrm{P}]+\mathrm{H}_{2} \mathrm{O} \rightleftharpoons \mathrm{NaOH}+\mathrm{H}$ & $\mathrm{k}=2.2 \times 10^{-6} \exp (-21900 / T)$ & $3 \times 10^{3} \mathrm{~T}^{-1.293} \exp (-24129 / \mathrm{T})$ & Est as $\mathrm{Na}[3 \mathrm{P}]$ \\
\hline R73 & $\mathrm{Na}[4 \mathrm{P}]+\mathrm{HCl} \rightleftharpoons \mathrm{NaCl}+\mathrm{H}$ & $\mathrm{k}=4 . \times 10^{-12} \mathrm{~T}^{0.50}$ & $3.825 \times 10^{1} \mathrm{~T}^{-0.646} \exp (-40805 / \mathrm{T})$ & Weiss et al. (1988) \\
\hline R74 & $\mathrm{Na}[3 \mathrm{P}]+\mathrm{Na}[4 \mathrm{P}] \rightarrow \mathrm{Na}^{+}+\mathrm{Na}+\mathrm{e}^{-}$ & $\mathrm{k}=1 . \times 10^{-9}$ & & Carré et al. (1984) \\
\hline R75 & $2 \mathrm{Na}[3 \mathrm{P}] \rightarrow \mathrm{Na}_{2}^{+}+\mathrm{e}^{-}$ & $\mathrm{k}=2.15 \times 10^{-13} \mathrm{~T}^{0.50}$ & & Geltman (1988) \\
\hline R76 & $\mathrm{Na}[3 \mathrm{P}]+\mathrm{Na}[3 \mathrm{D}] \rightarrow \mathrm{Na}_{2}^{+}+\mathrm{e}^{-}$ & $\mathrm{k}=4.7 \times 10^{-12} \mathrm{~T}^{0.50}$ & & Babenko et al. (1995) \\
\hline R77 & $\mathrm{Na}_{2}^{+}+\mathrm{e}^{-} \rightarrow \mathrm{Na}+\mathrm{Na}[3 \mathrm{P}]$ & $\mathrm{k}=1 . \times 10^{-8}$ & & Est \\
\hline R78 & $\mathrm{Na}[4 \mathrm{P}]+\mathrm{K}[4 \mathrm{P}] \rightarrow \mathrm{Na}+\mathrm{K}^{+}+\mathrm{e}^{-}$ & $\mathrm{k}=1 . \times 10^{-9}$ & & Carré et al. (1984) \\
\hline R79 & $\mathrm{Na}[3 \mathrm{P}]+\mathrm{K}[5 \mathrm{P}] \rightarrow \mathrm{Na}+\mathrm{K}^{+}+\mathrm{e}^{-}$ & $\mathrm{k}=1 . \times 10^{-9}$ & & Carré et al. (1984) \\
\hline $\mathrm{R} 80$ & $\mathrm{Na}[4 \mathrm{P}]+\mathrm{K}[5 \mathrm{P}] \rightarrow \mathrm{Na}+\mathrm{K}^{+}+\mathrm{e}^{-}$ & $\mathrm{k}=1 . \times 10^{-9}$ & & Carré et al. (1984) \\
\hline $\mathrm{R} 81$ & $2 \mathrm{~K}[4 \mathrm{P}] \rightarrow \mathrm{K}_{2}^{+}+\mathrm{e}^{-}$ & $\mathrm{k}=5 . \times 10^{-13} \mathrm{~T}^{0.50}$ & & Klucharev et al. (1977) \\
\hline $\mathrm{R} 82$ & $2 \mathrm{~K}[4 \mathrm{P}] \rightarrow \mathrm{K}+\mathrm{K}[5 \mathrm{P}]$ & $\mathrm{k}=2 . \times 10^{-13} \mathrm{~T}^{0.50}$ & & Namiotka et al. (1997) \\
\hline $\mathrm{R} 83$ & $\mathrm{~K}[4 \mathrm{P}]+\mathrm{K}[5 \mathrm{P}] \rightarrow \mathrm{K}+\mathrm{K}^{+}+\mathrm{e}^{-}$ & $\mathrm{k}=1 . \times 10^{-9}$ & & Carré et al. (1984) \\
\hline $\mathrm{R} 84$ & $\mathrm{~K}_{2}^{+}+\mathrm{e}^{-} \rightarrow \mathrm{K}+\mathrm{K}[4 \mathrm{P}]$ & $\mathrm{k}=1 . \times 10^{-8}$ & & Est \\
\hline R85 & $\mathrm{K}[4 \mathrm{P}] \rightleftharpoons \mathrm{K}+h \nu$ & $\mathrm{k}=3.8 \times 10^{7}$ & & Kramida et al. (2013) \\
\hline $\mathrm{R} 86$ & $\mathrm{~K}[5 \mathrm{P}] \rightleftharpoons \mathrm{K}+h \nu$ & $\mathrm{k}=1.1 \times 10^{6}$ & & Kramida et al. (2013) \\
\hline $\mathrm{R} 87$ & $\mathrm{~K}[3 \mathrm{D}] \rightleftharpoons \mathrm{K}[4 \mathrm{P}]+h \nu$ & $\mathrm{k}=1.74 \times 10^{7}$ & & Kramida et al. (2013) \\
\hline $\mathrm{R} 88$ & $\mathrm{~K}[5 \mathrm{~S}] \rightleftharpoons \mathrm{K}[4 \mathrm{P}]+h \nu$ & $\mathrm{k}=1.18 \times 10^{7}$ & & Kramida et al. (2013) \\
\hline R89 & $\mathrm{K}[5 \mathrm{P}] \rightleftharpoons \mathrm{K}[5 \mathrm{~S}]+h \nu$ & $\mathrm{k}=4.6 \times 10^{6}$ & & Kramida et al. (2013) \\
\hline R90 & $\mathrm{K}[5 \mathrm{P}] \rightleftharpoons \mathrm{K}[3 \mathrm{D}]+h \nu$ & $\mathrm{k}=1 . \times 10^{6}$ & & Kramida et al. (2013) \\
\hline R92 & $\mathrm{K}[4 \mathrm{P}]+\mathrm{M} \rightleftharpoons \mathrm{K}+\mathrm{M}$ & $\mathrm{k}=3.0 \times 10^{-12} \mathrm{~T}^{-0.50}$ & 1. $\exp (-18594 / \mathrm{T})$ & Earl and Herm (1974) \\
\hline R94 & $\mathrm{K}[4 \mathrm{P}]+\mathrm{HCl} \rightleftharpoons \mathrm{KCl}+\mathrm{H}$ & $\mathrm{k}=1.6 \times 10^{-12} \mathrm{~T}^{0.50}$ & $2.707 \times 10^{+01} \mathrm{~T}^{-0.648} \exp (-30060 / \mathrm{T})$ & Earl and Herm (1974) \\
\hline R96 & $\mathrm{K}[5 \mathrm{~S}]+\mathrm{M} \rightleftharpoons \mathrm{K}[4 \mathrm{P}]+\mathrm{M}$ & $\mathrm{k}=3.0 \times 10^{-12} \mathrm{~T}^{-0.50}$ & 1. $\exp (-11448 / \mathrm{T})$ & Lin and Schilowitz (1984) \\
\hline R98 & $\mathrm{K}[3 \mathrm{D}]+\mathrm{M} \rightleftharpoons \mathrm{K}[4 \mathrm{P}]+\mathrm{M}$ & $\mathrm{k}=3.0 \times 10^{-12} \mathrm{~T}^{-0.50}$ & 1. $\exp (-12175 / \mathrm{T})$ & Lin and Schilowitz (1984) \\
\hline R100 & $\mathrm{K}[5 \mathrm{P}]+\mathrm{M} \rightleftharpoons \mathrm{K}[5 \mathrm{~S}]+\mathrm{M}$ & $\mathrm{k}=6 . \times 10^{-12} \mathrm{~T}^{-0.50}$ & 1. $\exp (-5264 / \mathrm{T})$ & Lin and Schilowitz (1984) \\
\hline R102 & $\mathrm{K}[5 \mathrm{P}]+\mathrm{M} \rightleftharpoons \mathrm{K}[3 \mathrm{D}]+\mathrm{M}$ & $\mathrm{k}=6 . \times 10^{-12} \mathrm{~T}^{-0.50}$ & 1. $\exp (-4537 / \mathrm{T})$ & Lin and Schilowitz (1984) \\
\hline R104 & $\mathrm{K}[5 \mathrm{P}]+\mathrm{H}_{2} \rightleftharpoons \mathrm{KH}+\mathrm{H}$ & $\mathrm{k}=1.2 \times 10^{-13} \mathrm{~T}^{0.50}$ & $7.54 \mathrm{~T}^{-0.678} \exp (-4936 / \mathrm{T})$ & Lin and Schilowitz (1984) \\
\hline R106 & $\mathrm{K}[5 \mathrm{P}]+\mathrm{H}_{2} \mathrm{O} \rightleftharpoons \mathrm{KOH}+\mathrm{H}$ & $\mathrm{k}=1 . \times 10^{-13} \exp (-20000 / T)$ & $1.789 \times 10^{3} \mathrm{~T}^{-1.277} \exp (-18757 / \mathrm{T})$ & Est as $\mathrm{Na}[3 \mathrm{P}]+\mathrm{H}_{2} \mathrm{O}$ \\
\hline R108 & $\mathrm{K}[5 \mathrm{P}]+\mathrm{HCl} \rightleftharpoons \mathrm{KCl}+\mathrm{H}$ & $\mathrm{k}=4 . \times 10^{-12} \mathrm{~T}^{0.50}$ & $2.707 \times 10^{1} \mathrm{~T}^{-0.648} \exp (-34596 / \mathrm{T})$ & Est as $\mathrm{Na}[3 \mathrm{P}]$ \\
\hline R109 & $\mathrm{K}[4 \mathrm{P}, 5 \mathrm{~S}, 3 \mathrm{D}, 5 \mathrm{P}]+h \nu \rightarrow \mathrm{K}^{+}+\mathrm{e}^{-}$ & & & \\
\hline
\end{tabular}


to $\mathrm{Na}[3 \mathrm{P}], \mathrm{Na}[4 \mathrm{P}], \mathrm{K}[4 \mathrm{P}]$ and $\mathrm{K}[5 \mathrm{P}]$. Each of these states is practically a doublet, but we are treating them as a single state in the photochemical calculations, i.e. we assume they have the same reactivity and radiative lifetimes. Although, different reactivities between the two terms have been observed for higher laying states, such effects are small and they will not affect our conclusions regarding the importance of the excited states chemistry on the overall abundance of the alkalis. Higher excited states of ${ }^{2} \mathrm{P}$ symmetry can also be excited from the ground state, but the required shorter energy photons will be absorbed by other species higher up in the atmosphere and the oscillator strengths for these transitions decrease with increasing energy level. Further interaction of the first excited states with photons leads to the excitations of the $[4 \mathrm{~S}]$ and $[3 \mathrm{D}]$ states of $\mathrm{Na}$ and the $[5 \mathrm{~S}]$ and $[3 \mathrm{D}]$ states of $\mathrm{K}$, which in their turn excite to the $\mathrm{Na}[4 \mathrm{P}]$ and $\mathrm{K}[5 \mathrm{P}]$ states, respectively (Fig. 12). These are the four states we consider for each atom. We only consider excited states of the neutral alkali atoms, since excited states of the ions are separated by tens of eVs from the corresponding ground ion states.

We investigate in our calculations the different chemical reactivity of the excited states towards $\mathrm{H}_{2}, \mathrm{H}_{2} \mathrm{O}$, and $\mathrm{HCl}$. These reactions can lead to the formation of molecular complexes that could reduce the atomic abundances. To avoid interrupting the flow of the text we moved the details of this study to the appendix. Our results demonstrate that these processes can not significantly affect the atomic abundances of $\mathrm{Na}$ and $\mathrm{K}$. In addition, the simulated non-LTE populations are not large enough to affect the transit depth observations. Our assumed atmospheric conditions and composition are different from those used by Barman et al. (2002), which is probably the reason for the different conclusions derived between the two studies about the efficiency of the non-LTE processes. Nevertheless, we note that the overall effect of the extended chemistry processes we include (see below) can affect the upper atmosphere abundances of the alkali atoms.

\subsection{Ion chemistry}

Excited alkali metals can further contribute to the ionization of the atmosphere. Direct photoionization from the radiation field

$$
\mathrm{Na}^{*}+h \mathrm{v} \rightarrow \mathrm{Na}^{+}+\mathrm{e}^{-},
$$

is more efficient for the excited states since they are closer to the ionization potential, i.e. the ionization cross sections of the excited states extend to longer wavelengths than those of the ground state. Therefore their ionization rates can be significant since the stellar fluxes increase with increasing wavelength in this part of the spectrum. We calculated the photo-ionization cross section of the excited states following the analytical description from Zhang and Shneider (2010) that is consistent with available measurements (Wippel et al. 2001; Amin et al. 2005).

Energy pooling reactions can also populate higher excited levels that can photo-ionize faster. For example, Huennekens and Gallagher (1983b) have measured reaction rates for the process:

$$
2 \mathrm{Na}^{*} \rightarrow \mathrm{Na}[\mathrm{nl}]+\mathrm{Na}
$$

and found cross sections of 23 and $16 \AA^{2}$ for production of the $[4 \mathrm{D}]$ and $[5 \mathrm{~S}]$ states, respectively, from collision of two $\mathrm{Na}[3 \mathrm{P}]$ atoms at $600 \mathrm{~K}$. Theoretical calculations that are consistent with these measurements show that at higher temperatures the cross section for the formation of the $[4 \mathrm{~S}]$ state should increase to $\sim 40 \AA^{2}$ at 1000 $\mathrm{K}$, while that for $[5 \mathrm{~S}]$ should decrease but less steeply Geltman 1989). Energy pooling reaction for potassium where measured for collisions between $[4 \mathrm{P}]$ states to produce [5P], [6S], and [4D] states (Namiotka et al. 1997). According to our calculations, the loss rate of $\mathrm{Na}^{*}$ states due to energy pooling is small compared to the other processes we consider and since we did not include any higher states than the $\mathrm{Na}[4 \mathrm{P}]$, we did not include this mechanism. For potassium we included the energy pooling rate to the $\mathrm{K}[5 \mathrm{P}]$ state, but it has a minor role.

Other processes of direct ionization without a secondary photon involvement are those of Penning ionization:

$$
2 \mathrm{Na}^{*} \rightarrow \mathrm{Na}^{+}+\mathrm{Na}+\mathrm{e}^{-}
$$

and associative ionization:

$$
2 \mathrm{Na}^{*} \rightarrow \mathrm{Na}_{2}^{+}+\mathrm{e}^{-}
$$

These mechanisms occur for both sodium and potassium, but also for combinations of the two. Associative ionization for $\mathrm{Na}$ has been observed for the $[3 \mathrm{P}]$ states (Huennekens and Gallagher 1983a) as well as from collisions between [3P] and [3D] states (Babenko et al. 1995), while theoretical studies have demonstrated that the collision cross section for the associative ionization of two $[3 \mathrm{P}]$ atoms at $1000 \mathrm{~K}$ is more than an order of magnitude smaller than the corresponding energy pooling cross section (Geltman 1988). Associative ionization for potassium was measured by Klucharev et al. (1977) for two [4P] states, while Kimura and Saikan (1981) measured the penning and associative ionization of excited $\mathrm{Na}$ atoms with ground state $\mathrm{K}$ atoms and found that production of $\mathrm{K}^{+}$and $\mathrm{NaK}^{+}$is possible but occurs only for sodium energy levels above the [6S] state. Thus, associative ionization can not have an important contribution to the ionization of the $\mathrm{Na}$ and $\mathrm{K}$ for the conditions we simulate, as demonstrated also by the resulting profiles of the dimer ions (Figs. 20] \& 21).

Penning ionization is possible for collisions between the two resonant states of sodium and potassium:

$$
\begin{gathered}
\mathrm{Na}[3 \mathrm{P}]+\mathrm{Na}[4 \mathrm{P}] \rightarrow \mathrm{Na}+\mathrm{Na}^{+}+\mathrm{e}^{-} \\
\mathrm{K}[4 \mathrm{P}]+\mathrm{K}[5 \mathrm{P}] \rightarrow \mathrm{K}+\mathrm{K}^{+}+\mathrm{e}^{-},
\end{gathered}
$$

but also for the cross collision processes:

$$
\begin{aligned}
& \mathrm{Na}[4 \mathrm{P}]+\mathrm{K}[4 \mathrm{P}] \rightarrow \mathrm{Na}+\mathrm{K}^{+}+\mathrm{e}^{-} \\
& \mathrm{Na}[3 \mathrm{P}]+\mathrm{K}[5 \mathrm{P}] \rightarrow \mathrm{Na}+\mathrm{K}^{+}+\mathrm{e}^{-} \\
& \mathrm{Na}[4 \mathrm{P}]+\mathrm{K}[5 \mathrm{P}] \rightarrow \mathrm{Na}+\mathrm{K}^{+}+\mathrm{e}^{-}
\end{aligned}
$$

These processes have large reaction cross sections and we used an estimated reaction rate of $10^{-9} \mathrm{~cm}^{3} \mathrm{~s}^{-1}$ based on the measurements for $\mathrm{Na}[3 \mathrm{P}]+\mathrm{Na}[5 \mathrm{~S}]$ collisions from Carré et al. (1984). Similar processes for the other states are also possible, but the populations of the resonant states are much larger and have the highest contribution to the alkali ionization through this pathway.

Inclusion of these processes does not affect the densities of $\mathrm{Na}$ and $\mathrm{K}$ below 1 mbar, and therefore does not change 
our transit depth results. Any change in the profiles occurs only in the upper atmosphere and could be only be observationally detected by looking at the cores of the $\mathrm{Na}$ and $\mathrm{K}$ lines that are probing this region of the atmosphere. Our calculations show that inclusion of the extended chemistry reduces the differential transit depth at the $\mathrm{Na}$ line by $\sim 15 \%$ at the smallest bands widths around the line cores.

\subsection{Further notes $\&$ conclusions}

We finally add a few notes on the ground state ion chemistry. Radiative recombination of atomic ions usually leaves the neutral atom in an excited state from which it subsequently de-excites. We have not found any state specific information for the recombination of $\mathrm{Na}$ and $\mathrm{K}$ ions, thus we assumed that the produced neutrals are at the first excited states $(\mathrm{Na}[3 \mathrm{P}]$ and $\mathrm{K}[4 \mathrm{P}])$. Assuming recombination to the $\mathrm{Na}[4 \mathrm{P}]$ and $\mathrm{K}[5 \mathrm{P}]$ states did not change significantly our results. In the 3 -body recombination the excess energy is captured by the collision partner and the produced atoms should be on their ground state. We note that the released energy from the recombination of sodium $(5.14 \mathrm{eV})$ is larger than the binding energy of the $\mathrm{H}_{2}$ molecule $(4.52 \mathrm{eV})$, thus the 3 -body recombination could lead to the dissociation of $\mathrm{H}_{2}$ :

$$
\mathrm{Na}^{+}+\mathrm{e}^{-}+\mathrm{H}_{2} \rightarrow \mathrm{Na}+2 \mathrm{H},
$$

or at least to significant vibrational excitation. This process happens deep enough in the atmosphere for the atoms to rapidly recombine to $\mathrm{H}_{2}$. Thus, the $\mathrm{H}_{2}$ profile is not affected by the three body recombination. For potassium the ionization potential $(4.32 \mathrm{eV})$ is less than (although comparable with) the $\mathrm{H}_{2}$ binding energy and therefore the recombination will not dissociate the molecule although it will probably leave it in an excited vibrational level.

For the high pressure limit of three body recombination we assumed a rate of $10^{-7} \mathrm{~cm}^{3} \mathrm{~s}^{-1}$ due to lack of information. Typically, recombination rates depend inversely on the square root of $\mathrm{T}$, which means that the high pressure limit of the recombination rate close to the temperature minimum could be $\sim 50 \%$ smaller than deeper in the atmosphere. Our sensitivity test on this parameter show that we need to assume a high pressure limit of $10^{-11}$ before the change in the total recombination rate becomes significant to affect the profiles of $\mathrm{Na}$ and $\mathrm{K}$. Thus, modifications to the assumed recombination rate do not seem capable to reduce the alkali abundances.

Due to their lower ionization potentials, charge transfer reactions of alkali atoms with other species are not efficient. Thus, the only charge transfer reactions to consider is:

$$
\mathrm{Na}^{+}+\mathrm{K} \leftrightarrow \mathrm{Na}+\mathrm{K}^{+}
$$

with the forward rate in this case favored by $0.82 \mathrm{eV}$. Another ionization mechanism is that of the ion-pair formation:

$$
\mathrm{Na}+\mathrm{X} \rightarrow \mathrm{Na}^{+}+\mathrm{X}^{-}
$$

where $\mathrm{X}$ is a species (atom or molecule) with a large enough electron affinity to make this reaction efficient at high temperatures. The best candidates in our case (in terms of abundance and electron affinity) are $\mathrm{H}, \mathrm{O}$, and $\mathrm{OH}$ with electron affinities of $0.75,1.46$, and 1.83 $\mathrm{eV}$, respectively, which means that the barriers for the ion-pairs formation will be $4.39,3.68$, and $3.31 \mathrm{eV}$. The overall effect of these processes on the alkali atoms is small. It is interesting to note that $\mathrm{Na}$ and $\mathrm{K}$ also form stable negative ions with electron affinities of 0.55 and $0.50 \mathrm{eV}$, respectively. In this case the corresponding ion pair reactions should be limited among the alkali atoms:

$$
\begin{aligned}
\mathrm{Na}+\mathrm{Na} & \rightarrow \mathrm{Na}^{+}+\mathrm{Na}^{-} \\
\mathrm{K}+\mathrm{K} & \rightarrow \mathrm{K}^{+}+\mathrm{K}^{-} \\
\mathrm{Na}+\mathrm{K} & \rightarrow \mathrm{Na}^{+}+\mathrm{K}^{-} \\
\mathrm{Na}+\mathrm{K} & \rightarrow \mathrm{Na}^{-}+\mathrm{K}^{+}
\end{aligned}
$$

The reduction in the involved energy barriers for these reactions is not as large as for the ion-pair formation processes with other species, and thus these mechanisms are not efficient. In any case, the ion-pair recombination (reverse of the above processes) is a barrierless reaction, therefore the net contribution of the ion-pair mechanism will be important only at very high temperatures and does not affect our conclusions for HD 209458 b.

Associative ionization of sodium atoms with water molecules, although important in the atmospheres of our solar system, is not significant for the high temperature exoplanet atmosphere as the bond energy is small $(\sim 1 \mathrm{eV})$ and the formed ligands would not survive in the atmosphere in significant abundances Gilligan et al. 2001). This effect is already demonstrated for the alkali dimers described above. The same conclusion also applies to the formation of clusters of alkali ions with $\mathrm{H}_{2} \mathrm{O}$ or $\mathrm{H}_{2}$, which can reduce the ion abundances due to their faster recombination rates. The high atmospheric temperatures would inhibit a significant population of the clusters, which even if they were possible would only make the agreement with the observations worse since the population of neutral atoms would increase. Therefore, we do not explicitly follow these processes in our calculations.

Finally we investigated the role of the atmospheric mixing on the profiles of $\mathrm{Na}$ and $\mathrm{K}$. Specifically we assumed a eddy mixing profile that is ten times smaller than the nominal profile we use. The resulting mixing ratios are not significantly different from the previous results in the region probed by the transit observations. As a result, our attempts to match the $\mathrm{Na}$ differential transit depth and explain the non-detection of $\mathrm{K}$ with our photochemical model are not successful.

\section{THE CASE OF XO-2 b}

Our calculations based on pure photochemical processes can not reproduce all available observations for the case of HD 209458 b. As we demonstrate in this section, however, our model can match the observations of both potassium and sodium absorption on XO-2 b (Sing et al., 2010, 2012). This is interesting because it demonstrates that the atmosphere of $\mathrm{XO}-2 \mathrm{~b}$ may be fundamentally different from HD209458b where we cannot explain the STIS observations with our clear atmosphere model.

Apart from the physical parameters of this system (planet radius, mass, and distance from parent star), we need to re-evaluate the temperature profile and stel- 


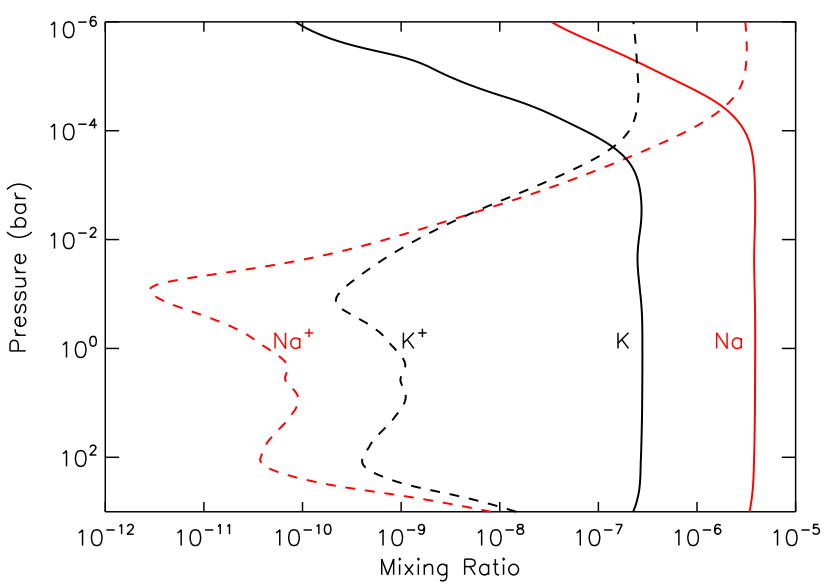

FIG. 13.- Mixing ratio profiles of $\mathrm{Na}$ (red) and $\mathrm{K}$ (blue) in the atmosphere of $\mathrm{XO}-2 \mathrm{~b}$. Solid lines present the neutral atoms and dashed the ions.

lar flux in order to perform the photochemistry simulation. For the stellar spectrum we used results from the PHOENIX model (Husser et al. 2013) for a star with $\log (\mathrm{g})$ and effective temperature of XO-2 (www.exoplanet.eu) that provides the photospheric contribution $(\lambda>150 \mathrm{~nm})$. We estimated the chromospheric contribution at shorter wavelengths from the HD 209458 stellar spectrum and from measurements of the ratios of FUV and EUV luminosity to the bolometric luminosity for XO-2 b, with updated values from Shkolnik (2013). These measurements indicate that the chromospheric flux of XO-2 is weaker than that of HD 209458. Therefore, we performed calculations assuming chromospheric fluxes with the same structure as for the HD 209458 spectrum but reduced by factors of 2 and 4 . Photons at chromospheric wavelengths are absorbed at high altitudes (see Fig. 3) and based on our calculations any small modifications induced on the $\mathrm{Na}$ and $\mathrm{K}$ profiles are limited to altitudes above the $1 \mu$ bar level. Thus, variations in the chromospheric flux will not affect our conclusions for the deep-atmosphere alkali abundances. For the temperature profile, we note that the orbital distance of XO-2 b (0.03 au) leads to a similar skin temperature as for HD 209458 b (see dotted lines in Fig. 2). Therefore we assumed the same (disk) temperature profile for this calculation.

Our resulting $\mathrm{Na}$ and $\mathrm{K}$ profiles for this atmosphere are similar to the profiles we calculate for HD 209458 b (Fig. 13). The comparison with the transit observations for this case reveals a very good agreement with the $\mathrm{R}_{p} / \mathrm{R}_{\text {star }}$ measurements at the potassium line (Fig. 14). In addition our simulated broadband (4066$7590 \AA) \mathrm{R}_{p} / \mathrm{R}_{\text {star }}$ for the Na line is 0.1054 that is in excellent agreement with the measured value of $0.1052 \pm 0.0011$ from Sing et al. (2012), while the differential planet radius around the sodium core is also consistent with the observations.

The contrast of the fit for XO-2 b with the calculations for HD $209458 \mathrm{~b}$ indicates that an explanation different from pure photochemical loss needs to be considered in order to explain the observations in the latter case. This explanation could be related to the presence of clouds in the atmosphere of HD 209458 b relative to a clear

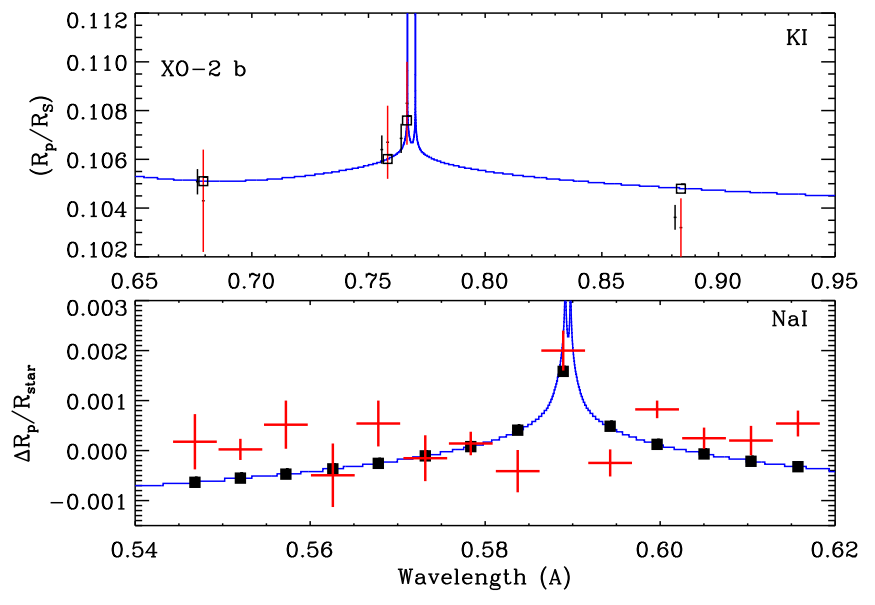

FIG. 14.- Top: Planet size calculation at the potassium line for the exoplanet $\mathrm{XO}-2 \mathrm{~b}$. The blue line is the high resolution calculation and the squares correspond to the $\mathrm{R}_{p} / \mathrm{R}_{\text {star }}$ for the observed wavelength bins. Vertical red and black lines show the observed planet size range at four wavelengths based on the Sing et al. (2010) observations. The two sets of observations correspond to analysis based on the individual lines or combined analysis of all lines. Botttom: Comparison of the differential planet radius at the sodium core based on the calculations in blue, and the observations by Sing et al. (2012) in red. The error bars in the measurements correspond to the 1 sigma level and the wavelength bins used have a width of $50 \AA$. The black squares correspond to the model results at the resolution of the observations.

atmosphere in the case of XO-2 b, or a different elemental abundance of the alkali atoms.

In general, recent observations of different extrasolar planets point to variety of different scenarios. Sometimes both $\mathrm{Na}$ and $\mathrm{K}$ are detected, sometimes only $\mathrm{Na}$ is detected and sometimes only $\mathrm{K}$ is detected (G. Ballester, personal communication). In light of these observations we discuss the potential processes that could explain this variety below.

\section{DISCUSSION}

We discuss here possible scenarios that could help us interpret the $\mathrm{Na}$ and $\mathrm{K}$ observations in the atmosphere of HD 209458 b. These include modifications in the atmospheric temperature profile, the presence of clouds/haze, and the possibly different abundances of these elements relative to their solar values.

\subsection{Temperature effects}

One of the possible explanations proposed for the reduced $\mathrm{Na}$ transit depth and the lack of $\mathrm{K}$ is a sharp decrease in the temperature profile of HD $209458 \mathrm{~b}$ at pressures below $\sim 3$ mbar (Sing et al. 2008a) that would reduce the abundance of both elements due to their capture into condensates $\left(\mathrm{Na}_{2} \mathrm{~S}\right)$ or molecular structures $(\mathrm{KCl})$. Below this pressure level the temperature would have to remain high in order to reproduce the observed $\mathrm{Na}$ wing by Sing et al. (2008b). The vapor pressure curves for these conditions (see Fig. 22) indicate that temperatures lower by $\sim 300 \mathrm{~K}$ from the atmospheric skin temperature would be required for the alkali atoms to be significantly reduced. Such low temperatures however, are not supported by the observations.

At this point we need to make the distinction between day and night conditions. On one hand, the current cir- 


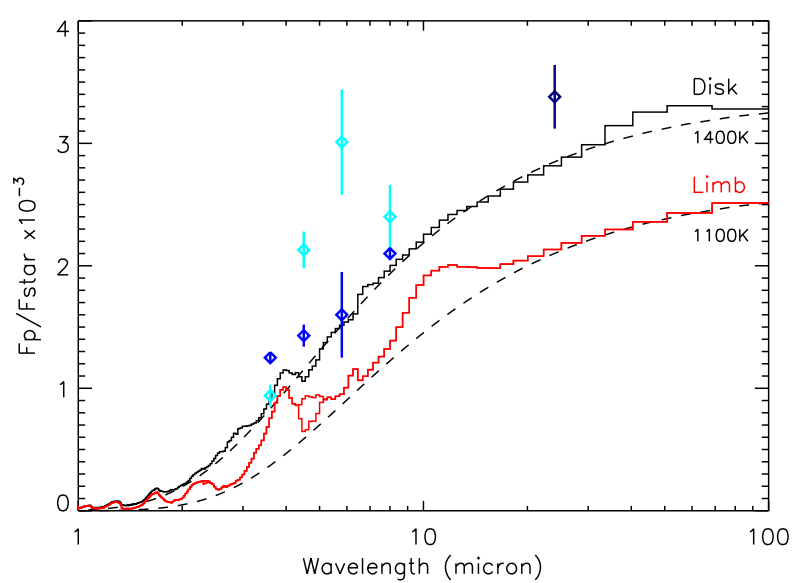

FIG. 15.- Ratio of planet to star fluxes for HD 209458 b. The black and red solid lines present the emissions assuming the disk and limb temperature profiles. The dashed lines present the anticipated flux ratios assuming black bodies of 1400 and $1100 \mathrm{~K}$, for the disk and limb respectively. Vertical bars with diamonds are the available observations from Knutson et al. (2008) in cyan, Diamond-Lowe et al. (2014) in blue, and Crossfield et al. (2012) in navy. The two red lines show the effect of $\mathrm{CO}$ addition close to $5 \mu \mathrm{m}$ (higher opacity brings the model closer to the black body solution).

culation models for HD 209458 b from which our temperature profiles for day-time disk and limb conditions are taken, suggest that strong zonal winds efficiently redistribute the heat flux from the day side to the night side (Showman et al. 2009). Thus, temperatures much lower than the skin temperature are not anticipated. On the other hand even if the night side is much colder than anticipated and the alkalis are captured in the form of condensates or molecules then the reduction in the observed transit depth would be only a factor of 2 , since the observations probe both the dusk and dawn terminators. Thus, a reduced temperature scenario does not seem able to explain the observed transit depth.

An argument against globally lower temperatures arises from the thermal emission of HD 209458 b (Fig. 15). With the assumed disk temperature profile and the calculated $\mathrm{H}_{2} \mathrm{O}$ and $\mathrm{CO}$ abundances from the photochemical model we derived a thermal emission spectrum that is consistent with the available observations from Spitzer. The latter include the initial observations by Knutson et al. (2008) between 3.6 and $8 \mu \mathrm{m}$, and the more recent observations at these wavelengths by Diamond-Lowe et al. (2014) that suggest a lower flux ratio, as well as the $24 \mu \mathrm{m}$ observations by Crossfield et al. (2012). The calculated spectrum indicates that a slightly warmer atmosphere would bring the thermal emission even closer to the observations. Hence, a colder atmosphere in order to the reduce the abundances of the alkali atoms, is not supported by these observations.

If we assume that the short wavelength slope in the transit depth observations is due to $\mathrm{H}_{2}$ Rayleigh scattering des Etangs et al. 2008), we can derive an estimate of the atmospheric temperature. Assuming different isothermal conditions we can investigate the variation of the simulated slope of the transit depth at short wavelengths against the observations (Fig. 16). Changing the atmospheric temperature, shifts the whole transit

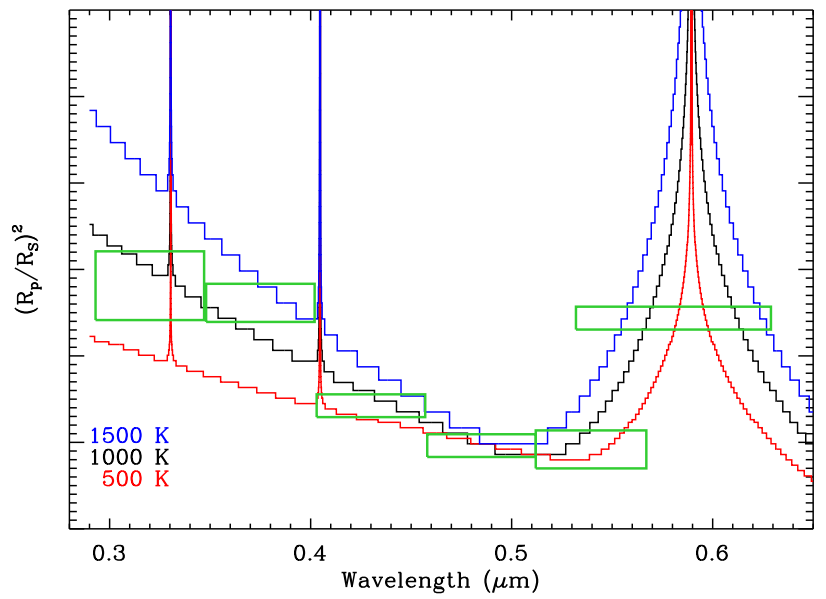

FIG. 16. - Comparison of the $\mathrm{H}_{2}$ Rayleigh slope of the simulated spectra at different temperatures with the slope of the HST/STIS observations (green boxes) from Deming et al. (2013).

depth curve due to the changes in the atmospheric scaleheight. Thus, in order to focus only on the variations of the Rayleigh slope we normalized our simulated transit spectra for different temperatures to $0.485 \mu \mathrm{m}$. For the comparison with the HST/STIS observations (which have small enough error bars to allow for this test) we scaled the simulated spectra so that they would result to the same transit depth at $0.485 \mu \mathrm{m}$. This comparison of the simulated slope with the observed slope demonstrates that temperatures close to $1000 \mathrm{~K}$ generate transit depth slopes that are in better agreement with the observed slope, compared to spectra generated for temperatures of 500 or $1500 \mathrm{~K}$. A best fit search assuming only $\mathrm{H}_{2}$ results in an isothermal profile of $\sim 1090 \mathrm{~K}$, which is very close to the isothermal part of the limb temperature profile we use (Fig. 2). Note that this conclusion does not depend on which data set of the broad band observations we use (Knutson et al. 2007; Deming et al. 2013), since we only compare the relative absorption depths at different wavelengths that are approximately the same for the two studies. Therefore, if $\mathrm{H}_{2}$ is responsible for the short wavelength slope of the observations, the temperature can not be low enough for $\mathrm{Na}$ and $\mathrm{K}$ to condense or be captured in molecular structures.

We note at this point that Vidal-Madjar et al. (2011) derived from the differential transit depth observations a cold enough temperature profile for HD 209458 b to allow for the loss of $\mathrm{Na}$ due to condensation. Their retrieval was based on the assumption of a constant with altitude Na profile, though, which our calculations show is not realistic above $\sim 1$ mbar (see Fig. 5 ). For a constant profile the temperature retrieval does not depend on the $\mathrm{Na}$ abundance, but this does not hold for a variable profile for which the differential transit depth has a different slope and magnitude (Fig. 17). Thus, a more reliable temperature retrieval based on these observations needs to consider the variation of the $\mathrm{Na}$ profile with altitude due to the photochemistry. Given the apparent inconsistency between the mid- and low-resolution transit depth observations, we are reluctant to perform such an analysis before a better understanding of the observations is possible.

A much warmer temperature scenario does not pro- 


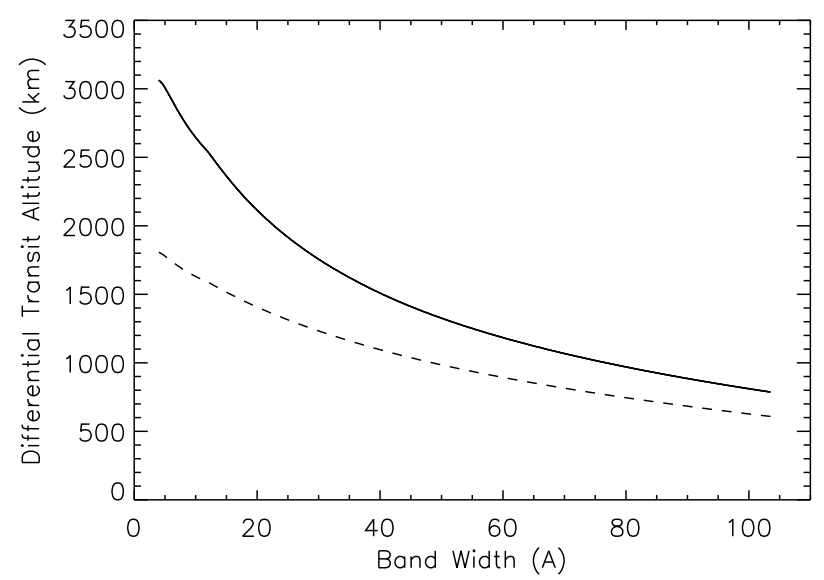

FIG. 17.- Differential transit altitudes derived for a hypothetical HD $209458 \mathrm{~b}$ atmosphere with a constant temperature of $1000 \mathrm{~K}$, and under different assumptions for the $\mathrm{Na}$ abundance and vertical profile. The thick solid line is a composite of 3 cases of constant $\mathrm{Na}$ mixing ratio and abundances of $0.1,1$, and 10 times solar. The dashed line corresponds to the case of the $\mathrm{Na}$ profile derived by our photochemical model (see Fig. [5).
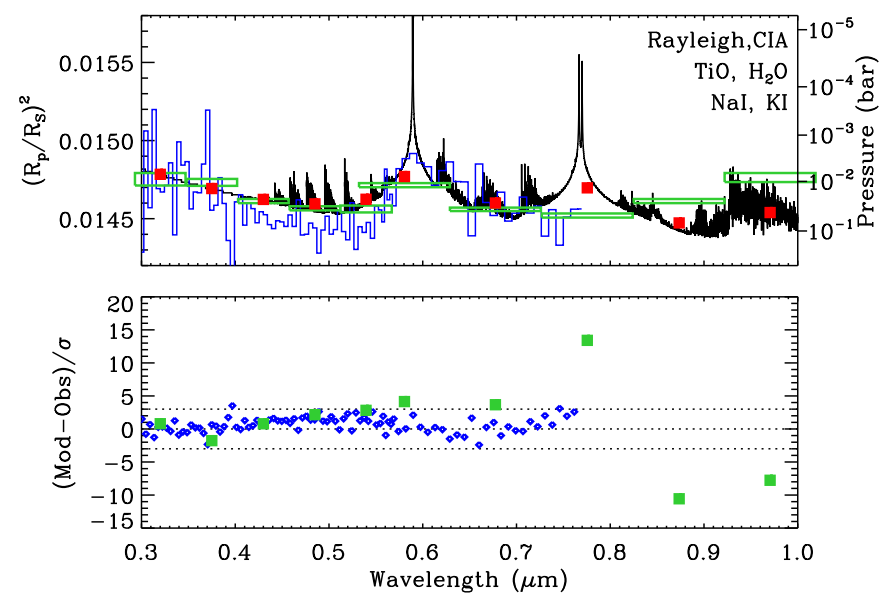

FIG. 18. - Same as Fig. [7 but with the inclusion of TiO, assuming a constant mixing ratio of $10^{-10}$.

vide a solution either; even if more exotic metals become available in the atmosphere such as $\mathrm{TiO}$ or $\mathrm{VO}$ (Fortney et al. 2003; Desert et al. 2008), their spectral signature is not large enough to cover the broad wings of the potassium line. For example, assuming a mixing ratio of $10^{-10}$ for $\mathrm{TiO}$, the transit depth at the sodium line center is larger than the observations, while the $\mathrm{K}$ wings are not affected (Fig. 18). Assuming a larger abundance $\left(10^{-9}\right)$, the transit depth at wavelengths between 0.4 and $0.75 \mu \mathrm{m}$ increases, but at shorter wavelengths it remains the same, as the $\mathrm{TiO}$ cross section sharply decreases for $\lambda<0.4 \mu \mathrm{m}$. Thus, a large $\mathrm{TiO}$ abundance is inconsistent with the observed broadband slope, if the latter is due to $\mathrm{H}_{2}$ Rayleigh scattering. If the slope is due to a different source of opacity, then a much higher temperature would be required in order to allow for large $\mathrm{TiO}$ abundance, enough to cover the K line and obscure the Na line. Such high temperatures would contradict the disk emissions, with which the temperature profile we use is consistent.

\subsection{Clouds and Hazes}

Another potential solution is that of a heterogenous opacity. In this scenario, the signatures of $\mathrm{Na}$ and $\mathrm{K}$ are reduced because a cloud or a photochemical haze is providing a spectrally homogeneous opacity that masks the alkali signature. As we demonstrated earlier this scenario, originally suggested as an explanation of the reduced differential transit depth around the $\mathrm{Na}$ doublet (Charbonneau et al. 2002), is not consistent with the subsequent observations (Ballester et al. 2007; Knutson et al. 2007; Sing et al. 2008b). On the other hand a reduced differential transit depth was retrieved in the subsequent observations from the high resolution spectra (Sing et al. 2008a) around the sodium core. One way to reconcile the two different conclusions derived from the same observations is to assume that the spectral region around the $\mathrm{Na}$ core is affected by another gaseous absorber with a relatively flat absorption spectrum. The problem with this scenario is that the $\mathrm{Na}$ doublet is one of the strongest absorption lines and in order to obscure its wings with another gaseous absorber, the abundance of the later would have to be too large. For example, TiO that has numerous lines around the Na core, would have to be present at a mixing ratio of $10^{-8}$ before it substantially affects the differential transit depth. In that case numerous absorption lines at shorter wavelengths would have an observable signature in the transit spectrum, and generate a different spectral slope than observed. Therefore, it is clear that the analysis of the low and high resolution observations from HST/STIS provide inconsistent results. The same problem appears in the comparison of the low resolution analysis results (Knutson et al. 2007; Deming et al. 2013) with the medium resolution spectra (Sing et al. 2008b), with the later demonstrating a more pronounced $\mathrm{Na}$ wing compared to the former. All these differences demonstrate the difficulties inherent in the analysis of these observations.

Nevertheless, if we momentarily neglect the differential transit observations (that are subject to higher uncertainty relative to the broadband results) a heterogeneous opacity can still be invoked to help understand the observations, provided that the haze or cloud structure can provide a short wavelength slope that is consistent with the observations. The probed pressures around the alkali lines indicate that this heterogenous opacity needs to be located close to the 10 mbar pressure level. It is important to keep in mind that we are observing the limb of the atmosphere. In this way the opacity of this cloud/haze does not have to be large in the vertical direction as long as the tangent opacity is consistent with the observations. Moreover, the consistency of the theoretical thermal emission spectrum with the observations (Fig. 15) implies that heterogeneous opacity is small and/or that the particle size is much smaller than $\sim 1 \mu \mathrm{m}$ in the probed region. Otherwise, scattering by the particles would modify the emitted radiation.

Heterogeneous opacities can also have an active photochemical role. For example, particles can accumulate free electrons in the atmosphere, and thereby decrease the recombination rates (e.g. Lavvas et al. 2013). This way the sodium and potassium abundances could be reduced deeper in the atmosphere. For the high temperatures of HD 209458 b, the large collision rates of the 
ions with the charged particles would limit the decrease in the recombination rate, though. Heterogenous reactions on the surfaces of the particles, however, could still affect the abundances of the alkali metals. All these processes require a dedicated study that will be presented in a future publication.

We should also note here that the case of heterogeneous opacity could also be consistent with the cases where only potassium is observed. In this case a low pressure opacity would be required (i.e. a photochemical haze rather than a cloud), in order to mask the $\mathrm{Na}$ line and with the appropriate slope in order to allow for the $\mathrm{K}$ line to be detected. The magnitude of the required slope could constrain the particle properties and be used to evaluate the feasibility of the haze scenario against other options. Moreover, the presence or not of line wings in the observations is a limiting factor for these scenarios, therefore high resolution observations, able to resolve these features are a requirement for future measurements.

\subsection{Elemental abundances}

Up to now we assumed solar elemental abundances in all of our calculations. The simplest method to reduce the $\mathrm{Na}$ and $\mathrm{K}$ transit depths is to assume that both elements have much different abundances in the atmosphere of HD 209458 b. This difference can be absolute or relative. If the ratio of the two elements is the same as solar but the absolute abundance is smaller, then we would have to reduce the abundances by a factor of 10 relative to the solar value in order to make the transit depth at the potassium line consistent with the observations (Fig. 19). In this case though, the corresponding reduction of the $\mathrm{Na}$ abundance would make the sodium wings much more narrow than what is suggested by the STIS observations analyzed by Sing et al. (2008a), and the overall transit depth would be much lower than the broadband absorption retrieved by Knutson et al. (2007) or Deming et al. (2013). Thus, a homogeneous decrease of the elemental abundances relative to the solar values is not consistent with the observations.

Is there though a reason to anticipate that the $\mathrm{Na} / \mathrm{K}$ ratio in HD $209458 \mathrm{~b}$ is similar to the solar ratio? Before answering this question we need to evaluate the degree at which we need to modify the assumed elemental abundances to bring the simulations in agreement with the observations. As discussed above, the solar abundance of $\mathrm{K}$ needs to be reduced by a factor of 10 to make its signature consistent with the transit depth spectrum from the Deming et al. (2013). Explaining the mid resolution HST/STIS observations, as presented in Desert et al. (2008), requires decreasing the solar $\mathrm{K}$ abundance by a factor of $\sim 50$ to bring the transit depth at the line core (the bin including the $\mathrm{K}$ line center in Desert et al. 2008) within 3 sigma of the observations. On the other hand, the solar Na abundance provides an acceptable fit to the sodium wing transit depth derived from the HST/STIS observations (neglecting the differential transit depth), implying that no major change is required for this element. These results imply that $\mathrm{Na} / \mathrm{K} \simeq 150-750$ in the atmosphere of HD $209458 \mathrm{~b}$, which is a substantial modification to the corresponding solar ratio, $(\mathrm{Na} / \mathrm{K})_{\odot} \simeq 15$. Thus, we can reformulate the above question to: is such a large abundance ratio realistic?

There are two approaches to answer this question. The first involves the actual spectrum of HD 209458, which demonstrates the presence of both $\mathrm{Na}$ and $\mathrm{K}$ as seen from ground based observations (e.g. Jensen et al. 2011). Although an evaluation of the abundance of these elements in the star's atmosphere is not available, the structure and magnitude of the spectral lines of the two elements imply that the abundance of $\mathrm{K}$ is not two orders of magnitude smaller than that of Na. Although not conclusive, this is the only constraint available for this system currently.

From a wider perspective we can address the question of the relative abundances of $\mathrm{Na}$ and $\mathrm{K}$ by observing the statistics of their ratio in different stars. Observations of very low metallicity massive stars, which are representative of the first stars formed in the universe, can provide us with information about the initial elemental abundance of heavy elements in the interstellar medium. Measurements of the sodium and potassium abundance ratios relative to magnesium in such stars of our galaxy give mean values of $[\mathrm{Na} / \mathrm{Mg}]=-0.8$ dex and $[\mathrm{K} / \mathrm{Mg}]=-0.3$ dex, with scatter of 0.14 and 0.09 , respectively (Andrievsky et al. 2010). Although these values suggest lower elemental abundances of the alkalis relative to the solar values (as anticipated for the early stages of the universe) they demonstrate that the $\mathrm{Na} / \mathrm{K}$ ratio ranges between 0.16 and 0.62 relative to the solar ratio. Observations of stars with similar properties as HD $209458 \mathrm{~b}$ and the sun show that $\mathrm{Na}$ and K abundances are close to the solar values (Shimansky et al. 2003; Adibekvan et al. 2012), while investigations of high metallicity A stars show that the variability of their alkali abundance is consistent with $\mathrm{Na} / \mathrm{K}<100$ (Takeda et al. 2012). Thus, a consistent picture arises from these observations that the large $\mathrm{Na} / \mathrm{K}$ values required to explain the observations are not likely, at least in stellar atmospheres. This conclusion though concerns only the stellar atmosphere rather than the planet.

Planetary atmospheres do not have to be of the same elemental abundance as the parent stars, as we well know from the solar system. It is important to note though that even if the elemental abundances in the atmosphere of HD 209458 b have been modified after the formation of the planet (e.g. due to migration in the stellar system followed by condensation and/or chemical modification), then we would expect $\mathrm{Na}$ and $\mathrm{K}$ to undergo the same changes, since they have very similar chemical behavior, as demonstrated above. In other words, we would not expect a preferential decrease of the potassium abundance over that of sodium during the evolution of the planetary atmosphere due to chemical processes only. Thus, any potential changes in the $\mathrm{Na} / \mathrm{K}$ ratio have to be traced back to the planet formation from the protoplanetary disk.

If the $\mathrm{Na} / \mathrm{K}$ ratio varied across the protoplanetary disk that gave rise to the planetary system, then modifications of the atmospheric ratio relative to the stellar value are possible. Variations of the abundances of different elements on the disk can occur as a result of turbulence, chemistry, and radiation pressure. Due to the large oscillator strengths of the resonant lines, $\mathrm{Na}$ and $\mathrm{K}$ are subject to strong radiative acceleration. This effect will be important only at disk regions that are optically thin and where the alkali metals are not ionized, and therefore will only affect a small part of the disk's mass. More- 

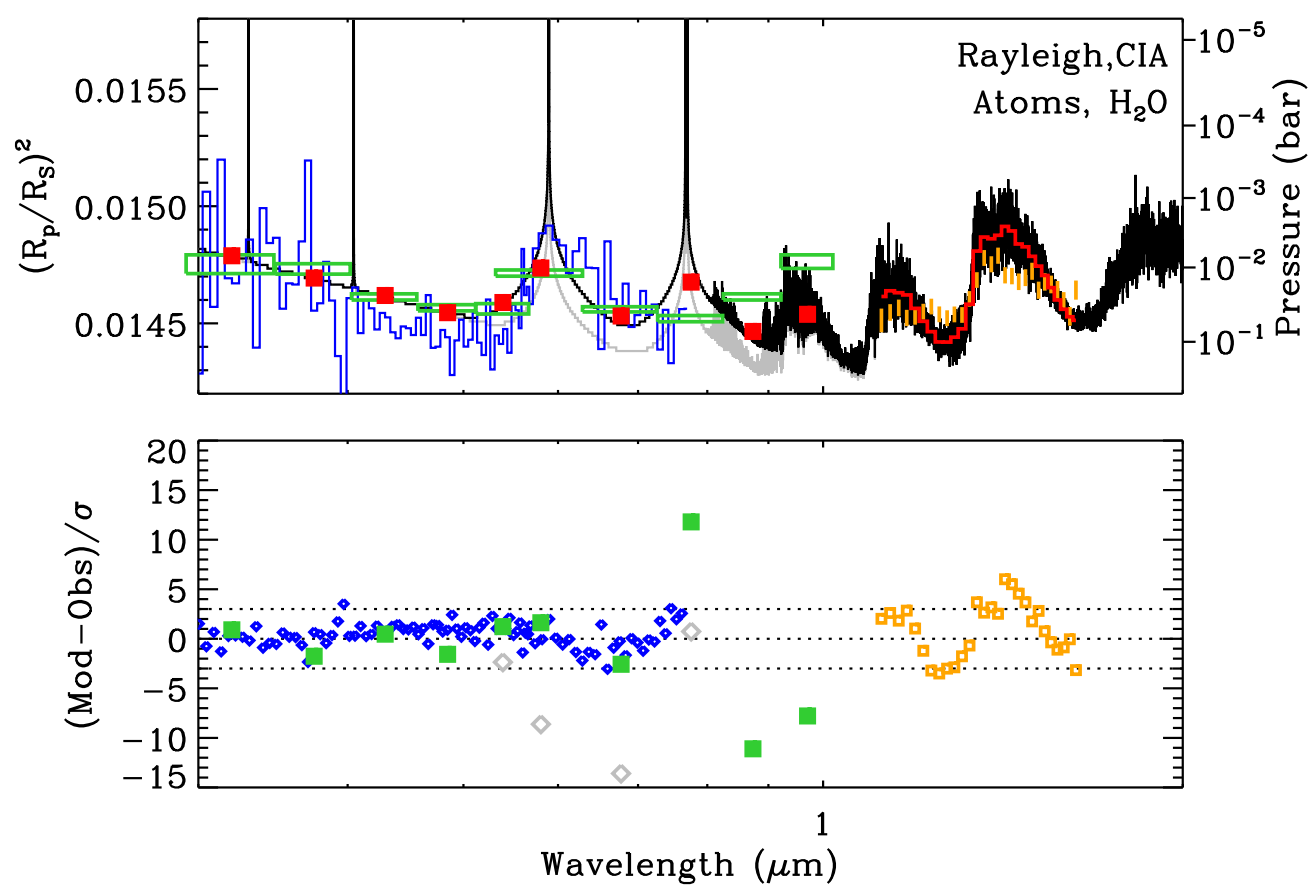

Fig. 19. - The same as Fig. 7 but zoomed in the region between 0.3 and $2 \mu \mathrm{m}$. The gray line in the top pager shows our model results in the case we divide the $\mathrm{Na}$ and $\mathrm{K}$ abundances by a factor of 10 , while the gray diamonds in the lower panel show how the model-observation differences for this case.

over, studies of this effect in debris disks demonstrate that, for low viscosity conditions, the $\mathrm{Na} / \mathrm{K}$ ratio (at 100 $\mathrm{AU})$ decreases relative to the solar value, contrary to the required modification in our case (Xie et al. 2013).

On the other hand, models coupling transport with photochemistry in protoplanetary disks (between 10 and $800 \mathrm{AU})$ demonstrate that the impact of turbulence varies among different gaseous species and ices, with the effect being more prominent on the latter component (Semenov and Wiebe 2011). This occurs as a result of the transport of ices/grains to regions of the disk where heterogeneous chemistry is more active (for the homogenous chemistry alone, no modifications were observed). The dominant condensates of alkalis in protoplanetary disks can be different from the condensates in planetary atmospheres, as the lack of cloud formation and stratification due to gravity, allows for the modification of condensates through interaction with other molecules. Thus, under thermochemical equilibrium $\mathrm{Na}$ and $\mathrm{K}$ should condense in the form of feldspar $\left(\mathrm{NaAlSi}_{3} \mathrm{O}_{8}, \mathrm{KAlSi}_{3} \mathrm{O}_{8}\right)$ in protoplanetary disks compared to $\mathrm{Na}_{2} \mathrm{~S}$ and $\mathrm{KCl}$ in planetary atmospheres (Lodders 2010a). Due to the limited life time of protoplanetary disks $(<10 \mathrm{Myr})$ and the variable lifetimes required for thermochemical equilibrium at different locations of the disk (due to variations in pressure and temperature) the abundance of the different components needs to be evaluated with detailed models that take into account the multiple processes involved. Unfortunately, a detailed study on the variation of $\mathrm{Na}$ and $\mathrm{K}$ over the protoplanetary disks is currently lacking. Any insight from observations is also limited. Spatially resolved spectra from the $\beta$-Pictoris debris disk demonstrate that $\mathrm{Na}$ is distributed over a large part for the disk, from 13 to 323 AU (Brandeker et al. 2004) but the corresponding distribution for $\mathrm{K}$ is unknown.

Nevertheless we can evaluate the protoplanetary disk conditions required to explain the HD 209458 b observations. The necessary $\mathrm{Na} / \mathrm{K}$ ratio retrieved from the observations implies that HD 209458 b should have emerged from a region of the disk that was deprived of $K$. This means that not only does $\mathrm{K}$ need to be lost from the gas phase but also its condensates or molecules need to be redistributed at a different radial location of the disk from where the planet is formed. Otherwise, gravitational heating (and the possible migration of the planet closer to the star) would have released the condensed alkalis back into the atmospherf. At the same time $\mathrm{Na}$ would have to remain unaltered by the processes affecting the $\mathrm{K}$ abundance. The current understanding of giant planet formation suggests that HD 209458 b was formed further away from the star ( $>1 \mathrm{AU})$ and subsequently migrated to its current location. Hence, both $\mathrm{Na}$ and $\mathrm{K}$ should be present in condensed form at the planet formation region, and would be subject to similar differentiation processes. Therefore, $\mathrm{Na}$ and $\mathrm{K}$ condensates should not have very different responses to the differentiation mechanisms. Moreover any differentiation process will have to occur in a short timescale, before the disk is accreted to the star, in order to allow for the migration of the planet at its current short orbital distance. Hence it is difficult to explain the observations of HD209458b based on chemical and physical processes in the protoplanetary disk at the time of planet formation.

Therefore, based on the expected primordial elemental abundances and alkali chemistry either in the protoplan-

\footnotetext{
4 We expect that $\mathrm{Na}$ and $\mathrm{K}$ captured in ices/rocks would be differentiated in the same way in the planet interior and subject to similar evaporation mechanisms.
} 
etary disk or the atmosphere of the planet, HD209458b should not have a $\mathrm{Na} / \mathrm{K}$ ratio that is substantially different from the solar ratio. While we cannot rule this possibility out unequivocally, it appears unlikely based on the above arguments.

\subsection{Observational issues}

Finally, there is one more aspect of the observations that requires some attention. The broad band observation on the red side of the $\mathrm{K}$ line demonstrate a significantly larger absorption than the model can produce. Absorption by $\mathrm{H}_{2} \mathrm{O}$ would be consistent with these observations if the temperature was higher than the profile we assume, and in this case the simulated spectrum would be in better agreement with the Spitzer/IRAC observations at 5.8 and $8 \mu \mathrm{m}$. In this case though the model would provide too much absorption for the other observational constraints from HST/WFC and Spitzer. These differences among the different observations could potentially be related to differences in the analysis applied, but could also be related to temporal changes in the properties of the star. Similar effects could be possible at shorter wavelengths and could affect the relative levels of the alkali lines relative to the slope at shorter wavelengths. Simultaneous and repeated observations of the whole spectrum are required in order to clarify all these observational issues.

\section{CONCLUSIONS}

Our photochemical calculations reveal that close-in exoplanets like HD 209458 b can have a significantly different ionospheric structure compared to the ionospheres of our solar system. From one hand, the proximity of the exoplanet to its parent star allows for very high ionization rates that result to large densities of ions and electrons (Fig. 6). On the other hand the most striking feature is the vertical distribution of these ions. From our solar system examples, ionospheres are dominantly found in the region of the thermosphere as the high energy photons $(\lambda \leq 1000 \AA)$ required to ionize the typical molecules found there $\left(\mathrm{H}_{2}, \mathrm{~N}_{2}, \mathrm{O}_{2}\right)$ can not penetrate at much deeper levels. The presence of heavier atoms with lower ionization potentials in the atmosphere of HD 209458 b, permits the ionization by longer wavelength photons $(\lambda \leq 3000 \AA)$, which are far more abundant.
As a result, ionization rates for the heavy atomic species are significantly higher and allow for large densities of ions and electrons in the lower part of the atmosphere (close to 0.1 mbar) compared to the corresponding densities generated due to thermal collisions. The potential implications of these high electron densities in the atmospheric properties are discussed in the accompanying paper (Koskinen et al. 2014).

We have also performed a thorough study on the possible mechanisms that could explain the observations of $\mathrm{Na}$ and $\mathrm{K}$ in the atmosphere of HD $209458 \mathrm{~b}$. In the past, the lack of a strong potassium signature along with the weak transit depth at the sodium core were attributed to either strong photo-ionization, a low temperature region in the atmosphere, the presence of clouds, or the different than solar elemental abundances of these elements in the atmosphere of HD 209458 b. We have demonstrated here that photochemistry alone can not explain the available observations, while we have also provided arguments against most of the other suggested scenarios. A much different temperature profile from that suggested by GCM models of this atmosphere are not supported by the observations, while a conveniently different elemental abundance is not likely based on observations of different stars, as well as of simulations of protoplanetary disks. The only case that appears more feasible is that of a heterogenous opacity from clouds or haze close to the 10 mbar pressure level that could screen the potassium absorption, but we showed that this scenario would not be consistent with the differential transit depth measurements around the Na core. Further studies on the role of clouds/aerosols on the heterogeneous chemistry of alkalis are required. On the other hand we have highlighted the inconsistencies among the different analyses of the same HST/STIS observations, which demonstrate the difficulties inherent in this process. Simultaneous and repeated observations of the whole spectrum (UV, visible and near IR) are required in order to resolve these inconsistencies and allow a better understanding for the abundance of alkali atoms in exoplanet atmospheres.

Notwithstanding the disagreement between the modeled and the implied by the observations abundance of $\mathrm{K}$, we note that our results for the atmospheric electron density are not modified for pressures below $\sim 1$ mbar, as this region is controlled by the photo-ionization of $\mathrm{Na}$ for which the model mixing ratio profile is in agreement with the observations.

\section{APPENDIX \\ EXCITED STATE CHEMISTRY}

The excited atoms can radiatively de-excite back to the ground state:

$$
\mathrm{Na}^{*} \rightarrow \mathrm{Na}+h \mathrm{v}
$$

(we use radiative de-excitation lifetimes from the NIST database, see Kramida et al. 2013), collide with another species and de-excite:

$$
\mathrm{Na}^{*}+\mathrm{M} \rightarrow \mathrm{Na}+\mathrm{M},
$$

or partake in a chemical reaction:

$$
\begin{gathered}
\mathrm{Na}^{*}+\mathrm{H}_{2} \mathrm{O} \rightarrow \mathrm{NaOH}+\mathrm{H} \\
\mathrm{Na}^{*}+\mathrm{H}_{2} \rightarrow \mathrm{NaH}+\mathrm{H} .
\end{gathered}
$$

In the above we used $\mathrm{Na}^{*}$ to describe any possible excited state of sodium, but we consider similar processes for potassium as well. The relative importance of each process is still under investigation and the available studies have 
demonstrated that the efficiency of each process does not depend only on the energy of the states involved, but also on the orbital configuration of the excited atoms (see review by Lin and Vetter 2002). In other words, Na and $\mathrm{K}$ can have a significantly different excited state chemistry.

The ro-vibrational distribution of the hydride molecules ( $\mathrm{NaH}$ and $\mathrm{KH}$ ) in these processes can provide important information about the reaction mechanisms involved. Theoretical calculations have identified two types of mechanisms, an insertion mechanism and a harpoon-type process. In the first case the excited atom attacks $\mathrm{H}_{2}$ by inserting itself between the two hydrogen atoms, while in the second case the alkali atom attacks the side of $\mathrm{H}_{2}$ in a co-linear mode, whereby the excited electron is inserted into the $\mathrm{H}_{2}$ molecule forming temporarily a negative ion that attracts the remaining alkali ion. The insertion mechanism leads eventually to the production of rotationally excited products, while the harpoon method favors the vibrational excitation of the resulting hydride molecule. These theoretical considerations are consistent with the experimentally derived rovibrational properties of the nascent hydride molecules (Bililign and Kleiber 1992; Bililign et al. 1992). Moreover they can explain the variation of the reaction properties for different states, as well as different alkali metals. For example, the insertion method is favorable for small atoms that suffer a minimal repulsion from $\mathrm{H}_{2}$ and is believed to describe the reaction mechanism for $\mathrm{Na}$ and Li, as well as the low laying states of K. For larger atoms (or excited states that have a larger effective size) the harpoon method is more favorable (see Chang et al. 2008, and references therein). Although these theoretical calculation can provide us with a qualitative picture of the alkali chemistry, the current level of detail can not provide us with quantitative description of the involved rates required for our calculations. Therefore, estimates of these rates need to rely on the available laboratory measurements.

The collisional deactivation rates for different excited states are usually reported in terms of a collision cross section, $\sigma$, between the excited atoms and the quenching species. The corresponding rate at temperature $\mathrm{T}$ can then be calculated from:

$$
k=\sqrt{\frac{8 k_{B} T}{\pi \mu}} \sigma
$$

where $\mu$ is the reduced mass of the colliding pair. For $\mathrm{Na}$ and $\mathrm{K}$ collisions with $\mathrm{H}_{2}$, this reduces too:

$$
k\left[\mathrm{~cm}^{3} \mathrm{~s}^{-1}\right]=10^{-12} \sqrt{\mathrm{T}} \sigma
$$

when $\sigma$ is given in $\AA^{2}$.

The collisional de-excitation of $\mathrm{Na}[3 \mathrm{P}]$ atoms by $\mathrm{H}_{2}$ was measured by Earl and Herm (1974) at high temperatures $(873 \mathrm{~K})$ who found a deactivation cross section of $10 \AA^{2}$. The formation of NaH from Na[3P] has a barrier $0.4-0.5 \mathrm{eV}$ (Motzkus et al. 1997) and non-reactive quenching was found to dominate through:

$$
\mathrm{Na}[3 \mathrm{P}]+\mathrm{H}_{2}(\nu=0) \rightarrow \mathrm{Na}+\mathrm{H}_{2}(\nu \geq 1)
$$

$\mathrm{Na}[3 \mathrm{P}]$ has enough energy to populate the first 3 vibrational levels of $\mathrm{H}_{2}$ and a subsequent reaction of the vibrationally excited hydrogen with other $\mathrm{Na}[3 \mathrm{P}]$ atoms can form $\mathrm{NaH}$ :

$$
\mathrm{Na}[3 \mathrm{P}]+\mathrm{H}_{2}(\nu \geq 1) \rightarrow \mathrm{NaH}+\mathrm{H}
$$

with a rate of $1.1 \times 10^{-9} \mathrm{~cm}^{-3} \mathrm{~s}^{-1}$ (Motzkus et al. 1998). Deactivation of $\mathrm{Na}[4 \mathrm{P}]$ state to the Na[3D] state was found to proceed with a cross section of $7 \AA^{2}$ at $700 \mathrm{~K}$ (Astruc et al. 1988), and deactivation of Na[4S] was observed to dominantly quench to $\mathrm{Na}[3 \mathrm{P}]$ with a cross section of $41.4 \AA^{2}$ (Astruc et al. 1986). In later studies, Kleiber et al. (1993) found that the reactive and non-reactive quenching channels in the collision of $\mathrm{Na}[4 \mathrm{P}]$ with $\mathrm{H}_{2}$ (at $470 \mathrm{~K}$ ) proceed with a ratio of $\sim 1$ and a total cross sections of $\sim 18 \AA^{2}$. Moreover, Motzkus et al. (1997) found that direct deactivation of $\mathrm{Na}[4 \mathrm{P}]$ to the ground state accompanied with the vibrational excitation of $\mathrm{H}_{2}$ is unlikely, and instead non-reactive quenching of $\mathrm{Na}[4 \mathrm{P}]$ proceeds to the $\mathrm{Na}[3 \mathrm{D}]$ state with the excess energy transformed to translation energy of each colliding member. Therefore, although both $\mathrm{Na}[3 \mathrm{P}]$ and $\mathrm{Na}[4 \mathrm{P}]$ eventually form NaH, the mechanisms involved are different (Motzkus et al. 1998). Based on these studies, we assume in our calculations non-reactive quenching of the $\mathrm{Na}[3 \mathrm{P}]$ state, and equal yields of reactive and non-reactive paths for the $\mathrm{Na}[4 \mathrm{P}]$ state. For the other sodium states we assume the same processes/rates as for the $\mathrm{Na}[4 \mathrm{P}]$ state, but we take into account the energy of each state in the calculation of the reverse reaction rates.

The collisional de-excitation of different states of potassium by $\mathrm{H}_{2}$ has been measured by Earl and Herm (1974) who derived total cross sections of 3 and $12 \AA^{2}$ for $\mathrm{K}[4 \mathrm{P}]$ and $\mathrm{K}[5 \mathrm{P}]$, respectively. Among the states we consider only $\mathrm{K}[5 \mathrm{P}]$ has enough energy for the chemical reaction. Early studies on the reactivity of this state have found that the relative contribution of the chemical pathway appears to be small (upper limit of $1 \%$ of total deactivation), while its quenching proceeds to the lower laying K[5S] and K[3D] states instead of the ground state K[4S] (Lin and Schilowitz 1984). Later studies where able to detect the formation of $\mathrm{KH}$ from $\mathrm{K}[5 \mathrm{P}]$ confirming the presence of the reaction channel (Liu and Lin 1996), as well as the non-reactive de-excitation pathways (Wong et al. 1999). Therefore, we assumed non-reactive quenching for the $\mathrm{K}[4 \mathrm{P}]$ state and a low yield (1\%) for reaction of the K[5P] state. For the other potassium states considered we assume the same processes/rates as for the K[4P] state.

For reactions with $\mathrm{H}_{2} \mathrm{O}$, Earl and Herm (1974) measured a total deactivation cross section of $3 \AA^{2}$ from the Na[3P] state, while Muller et al. (1980) derived a ratio for the chemical reaction rate to form $\mathrm{NaOH}$ from $\mathrm{Na}[3 \mathrm{P}] / \mathrm{Na}$ of 5350 

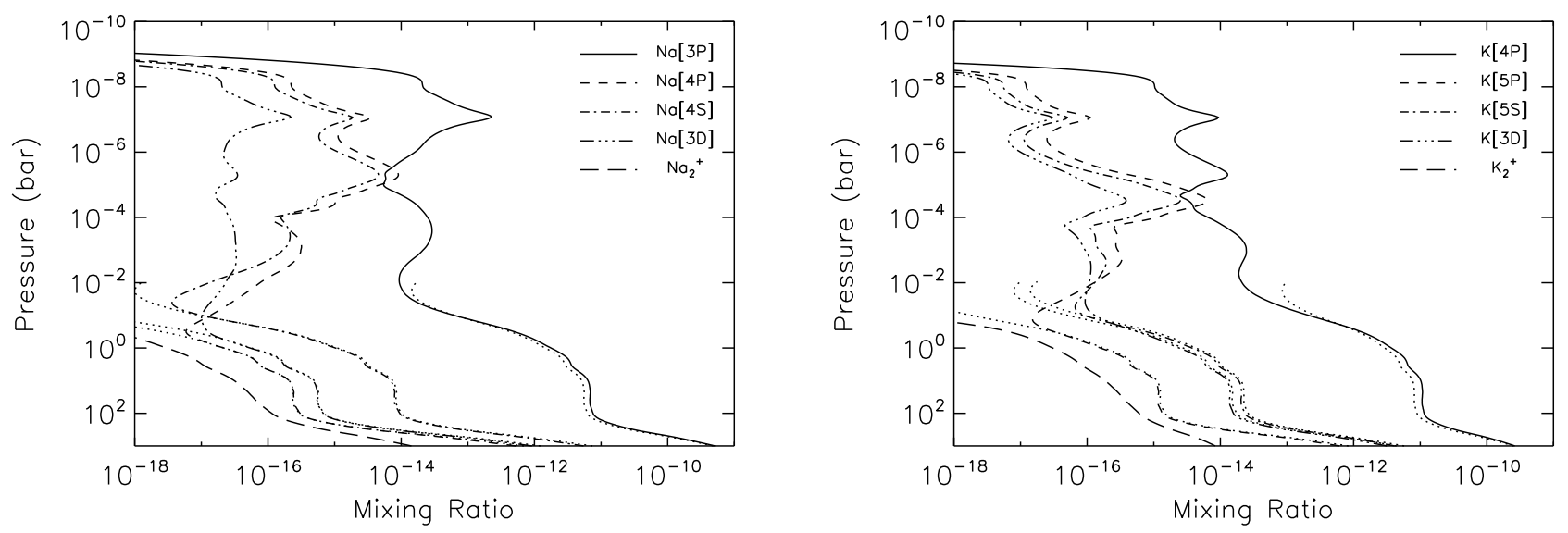

FIG. 20.- Mixing ratios of different excited states of Na and K. The dotted lines present the corresponding mixing ration assuming LTE conditions.
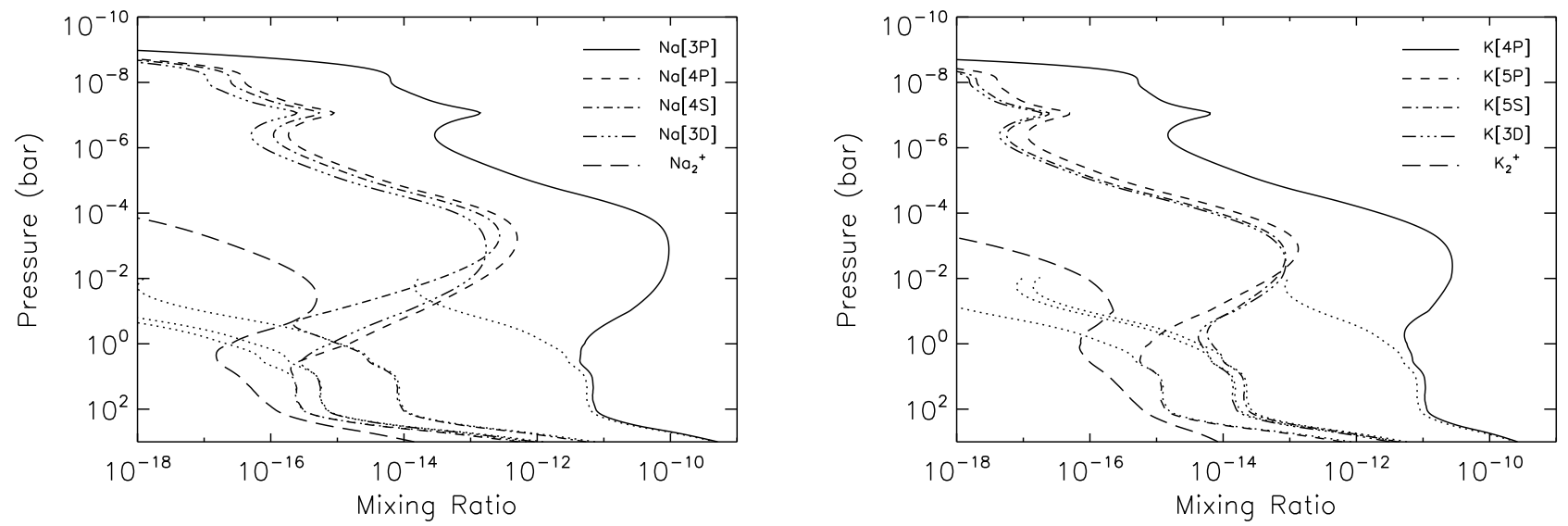

Fig. 21. - Mixing ratios of different excited states of $\mathrm{Na}$ and $\mathrm{K}$. The dotted lines present the corresponding mixing ration assuming LTE conditions. Here the attenuation of the stellar flux due to the resonance lines is not included.

at elevated temperature conditions $(1925 \mathrm{~K})$. Based on thermochemical consideration the reaction of $\mathrm{H}_{2} \mathrm{O}$ with $\mathrm{Na}[3 \mathrm{P}]$ is exothermic with a reaction constant that is more than five orders of magnitude larger than the reaction constant for $\mathrm{Na}$ at $\sim 2000 \mathrm{~K}$. Hence, the smaller value of the reaction ratio for the two states demonstrates that there is a reaction barrier involved. Reaction with higher energy states could have faster rates although we have not found any measurements for these processes. In lack of any information we have assumed the same rates as for the reaction of $\mathrm{Na}[3 \mathrm{P}]$, the latter estimated based on the observed ratio of $\mathrm{Na}[3 \mathrm{P}] / \mathrm{Na}$ reaction rates and the measured ground state reaction from Jensen (1982). For collisions of potassium with $\mathrm{H}_{2} \mathrm{O}$ the corresponding total cross sections are 2 and $84 \AA^{2}$ for K[4P] and K[5P], respectively (Earl and Herm 1974), and due to lack of information we assume a chemical reaction yield with the same ratio of $\mathrm{K}[5 \mathrm{P}] / \mathrm{K}$ rate as for sodium.

We have also considered the reactivity of the excited alkali atom states with $\mathrm{HCl}$. Weiss et al. (1988) have shown that the reactivity to form $\mathrm{NaCl}$, increases with increasing state energy for the states $3 \mathrm{~S}, 3 \mathrm{P}, 5 \mathrm{~S}$ and $4 \mathrm{D}$. They measured an average cross section of $\sim 4 \AA^{2}$ for the $\mathrm{Na}[3 \mathrm{P}]$ state, while for the other states we consider here we estimated a cross section of $10 \AA^{2}$ for reaction based on their results. Due to the lack of any measurements for potassium we have assumed the same cross sections to form $\mathrm{KCl}$ as for the corresponding sodium states.

The resulting profiles of excited states considered show that their populations are not large enough to affect the density of the alkali atoms (Fig. 201). For these calculations we assumed that once photons are absorbed by alkali metals in the resonance lines, they are irreversible lost. In reality, when collisional de-excitation is not efficient and atoms have the time to radiatively de-excite, the photons are re-emitted. In order to the evaluate the role of these photons we also considered the extreme scenario that the stellar flux is not attenuated by the alkali resonant lines, i.e. the photons are forwardly re-emitted. Under this extreme scenario the populations of the excited states are drastically increased (Fig. 21), but they still remain small enough not to affect the ground state populations of $\mathrm{Na}$ and $\mathrm{K}$. Therefore excited state chemistry can not help resolve the disagreement of the model with the observations. 
It is interesting to note that in laboratory experiments of $\mathrm{Na}$ resonant excitation a complete ionization of the gas was observed (Kushawaha and Leventhal 1982). This effect was attributed to a two step process: first a free electron was produced through the associative (or Penning) ionization process, and then followed by the super-elastic scattering of the electron by excited sodium atoms that eventually provides enough energy to the electrons to ionize all the sodium gas (Measures 1977). In our case though the presence of $\mathrm{H}_{2}$ will limit such effects as the excited alkali atoms will more easily transfer their energy to the vibrational levels of $\mathrm{H}_{2}$. What happens to the excited hydrogen molecules, though? We evaluated the role of vibrationally excited $\mathrm{H}_{2}$ in the formation of $\mathrm{NaH}$ through collisions with $\mathrm{Na}[3 \mathrm{P}]$ as described above. In this calculation we assumed that the vibrationally excited hydrogen is formed at the $\nu=2$ vibrational level from the reaction:

$$
\mathrm{Na}[3 \mathrm{P}]+\mathrm{H}_{2}(\nu=0) \leftrightarrow \mathrm{Na}+\mathrm{H}_{2}(\nu=2)
$$

The results show that for the conditions of the simulation $\mathrm{NaH}$ is not significantly affected by this process.

Another contribution to the excitation of alkali atoms as well as $\mathrm{H}_{2}$ could come from collisions with energetic electrons. In the region of the atmosphere we are interested the electron production is dominated by the photoionization of the alkali atoms. Collisions with $\mathrm{H}_{2}$ will gradually reduce the energy of these electrons to the thermal energy, but in this process the molecules will be vibrationally excited. Collision of electrons with the alkali atoms is also possible, but the excitation rate of the different states from this process will be small compared to the direct excitation by photons, as demonstrated by the comparison of the photo excitation rates to the photo-ionization rates for each atom (Fig. (4). The same argument also applies for the excitation of $\mathrm{H}_{2}$, since direct excitation by collisions with $\mathrm{Na}[3 \mathrm{P}]$ would be more efficient in the lower part of the atmosphere.

\section{ACKNOWLEDGMENTS}

We acknowledge fruitful discussions with Caitlin Griffith, Gilda Ballester, and Travis Barman. We thank Steven Klippenstein for fruitful discussions on the role of ion clusters, Bertrand Plez for providing the TiO line list, and our reviewer for the suggested improvements on the manuscript. PL acknowledges support by the French Programme National de Planétologie (PNP). TTK and RVY acknowledge support by the National Science Foundation (NSF) grant AST 1211514.

\section{REFERENCES}

Adibekyan, V. Z., Sousa, S. G., Santos, N. C., Delgado Mena, E., González Hernández, J. I., Israelian, G., Mayor, M., and Khachatryan, G. (2012). Chemical abundances of 1111 FGK stars from the HARPS GTO planet search program. Galactic stellar populations and planets. Astronomy and Astrophysics, 545:32.

Aldrovandi, S. M. V. and Pequignot, D. (1973). Radiative and Dielectronic Recombination Coefficients for Complex Ions. Astronomy and Astrophysics, 25:137-140.

Allard, N. F., Allard, F., Hauschildt, P. H., Kielkopf, J. F., and Machin, L. (2003). A new model for brown dwarf spectra including accurate unified line shape theory for the $\mathrm{Na} I$ and $\mathrm{K}$ I resonance line profiles. Astronomy and Astrophysics, 411:L473-L476.

Allison, T. C., Lynch, G. C., Truhlar, D. G., and Gordon, M. S. (1996). An Improved Potential Energy Surface for the H 2Cl System and Its Use for Calculations of Rate Coefficients and Kinetic Isotope Effects. The Journal of Physical Chemistry, 100(32): $13575-13587$.

Amin, N., Mahmood, S., Anwar-ul Haq, M., Riaz, M., and Baig, M. A. (2005). Measurement of the photoionization cross-section of the $3 \mathrm{p} 2 \mathrm{P} 1 / 2,3 / 2$ excited levels of sodium. The European Physical Journal D, 37(1):23-28.

Andrievsky, S. M., Spite, M., Korotin, S. A., Spite, F., Bonifacio, P., Cayrel, R., François, P., and Hill, V. (2010). Non-LTE abundances of $\mathrm{Mg}$ and $\mathrm{K}$ in extremely metal-poor stars and the evolution of $[\mathrm{O} / \mathrm{Mg}],[\mathrm{Na} / \mathrm{Mg}],[\mathrm{Al} / \mathrm{Mg}]$, and $[\mathrm{K} / \mathrm{Mg}]$ in the Milky Way. Astronomy and Astrophysics, 509:88.

Astruc, J. P., Barbe, R., Desfrancois, C., Lagreze, A., and Schermann, J. P. (1986). Direct laser excitation and quenching of the 4s state of sodium. Optics Communications, 57(3): $175-179$

Astruc, J. P., Desfrancois, C., Barbe, R., and Schermann, J. P. (1988). Intermultiplet and angular momentum transfers of excited sodium atoms in collisions with molecules. I.

Experiment. The Journal of Chemical Physics, 88(1):106-110.

Babenko, E., Tapalian, C., and Smith, W. W. (1995). Associative ionization in laser-excited sodium $3 \mathrm{p}+3 \mathrm{~d}$ collisions. Chemical Physics Letters, 244(1):121-126.

Ballester, G. E., Sing, D. K., and Herbert, F. (2007). The signature of hot hydrogen in the atmosphere of the extrasolar planet HD 209458b. Nature, 445(7127):511-514.

Barman, T. (2007). Identification of Absorption Features in an Extrasolar Planet Atmosphere. The Astrophysical Journal, 661(2):L191-L194.
Barman, T. S., Hauschildt, P. H., Schweitzer, A., Stancil, P. C., Baron, E., and Allard, F. (2002). Non-LTE Effects of Na I in the Atmosphere of HD 209458b. The Astrophysical Journal, 569(1):L51-L54.

Baulch, D. L., Duxbury, J., Grant, S. J., and Montague, D. C. (1981). Evaluated kinetic data for high temperature reactions. volume 4 homogeneous gas phase reactions of halogen- and cyanide- containing species. J. Phys. Chem. Ref. Data, 10:1-721.

Beaulieu, J. P., Kipping, D. M., Batista, V., Tinetti, G., Ribas, I., Carey, S., Noriega-Crespo, J. A., Griffith, C. A., Campanella, G., Dong, S., Tennyson, J., Barber, R. J., Deroo, P., Fossey, S. J., Liang, D., Swain, M. R., Yung, Y., and Allard, N. (2010). Water in the atmosphere of HD 209458b from 3.6-8 $\mu \mathrm{m}$ IRAC photometric observations in primary transit. Monthly Notices of the Royal Astronomical Society, 409(3):963-974.

Ben-Jaffel, L. and Sona Hosseini, S. (2010). On the Existence of Energetic Atoms in the Upper Atmosphere of Exoplanet HD209458b. The Astrophysical Journal, 709(2):1284-1296.

Bililign, S. and Kleiber, P. D. (1992). Nascent rotational quantum state distribution of $\mathrm{NaH}(\mathrm{NaD})$ from the reaction of $\mathrm{Na}^{*}(42 \mathrm{P})$ with H2, D2, and HD. The Journal of Chemical Physics, 96(1):213-217.

Bililign, S., Kleiber, P. D., Kearney, W. R., and Sando, K. M. (1992). Reactive collision dynamics of $\mathrm{Na}^{*}(42 \mathrm{P})+\mathrm{H} 2$ and $\mathrm{HD}$ : Experiment and theory. The Journal of Chemical Physics, 96(1):218-229.

Brandeker, A., Liseau, R., Olofsson, G., and Fridlund, M. (2004). The spatial structure of the ? Pictoris gas disk. Astronomy and Astrophysics, 413(2):681-691.

Brion, C. E., Dyck, M., and Cooper, G. (2005). Absolute photoabsorption cross-sections (oscillator strengths) for valence and inner shell excitations in hydrogen chloride, hydrogen bromide and hydrogen iodide. Journal Of Electron Spectroscopy And Related Phenomena, 144-147:127-130.

Brown, T. M. (2001). Transmission Spectra as Diagnostics of Extrasolar Giant Planet Atmospheres. The Astrophysical Journal, 553(2):1006-1026.

Brown, T. M., Charbonneau, D., Gilliland, R. L., Noyes, R. W., and Burrows, A. (2001). Hubble Space TelescopeTimeSeries Photometry of the Transiting Planet of HD 209458. The Astrophysical Journal, 552(2):699-709. 
Bryukov, M. G., Dellinger, B., and Knyazev, V. D. (2006). Kinetics of the Gas-Phase Reaction of $\mathrm{OH}$ with $\mathrm{HCl}$. Journal Of Physical Chemistry A, 110(3):936-943.

Burcat, A. and Ruscic, B. (2005). Third millennium ideal gas and condensed phase thermochemical database for combustion with updates from active thermochemical tables. Technical Report ANL 05/20, Argonne National Lab.

Carré, B., Spiess, G., Bizau, J. M., Dhez, P., Gérard, P., Wuilleumier, F., Keller, J. C., Le Gouet, J. L., Picque, J. L., Ederer, D. L., and Koch, P. M. (1984). Electron spectrometry study of associative and penning ionization in laser excited sodium vapor. Optics Communications, 52(1):29-34.

Chang, Y.-P., Hsiao, M.-K., Liu, D.-K., and Lin, K.-C. (2008). Rotational and vibrational state distributions of $\mathrm{NaH}$ in the reactions of $\mathrm{Na}(4[\sup 2] \mathrm{S}, 3[\sup 2] \mathrm{D}$, and6[sup 2]S) with $\mathrm{H}[$ sub 2]: Insertion versus harpoon-type mechanisms. The Journal of Chemical Physics, 128(23):234309.

Charbonneau, D., Brown, T. M., Noyes, R. W., and Gilliland, R. L. (2002). Detection of an Extrasolar Planet Atmosphere. The Astrophysical Journal, 568:377.

Crossfield, I. J. M., Knutson, H., Fortney, J., Showman, A. P. Cowan, N. B., and Deming, D. (2012). Spitzer/MIPS $24 \mu \mathrm{m}$ Observations of HD 209458b: Three Eclipses, Two and a Half Transits, and a Phase Curve Corrupted by Instrumental Sensitivity Variations. The Astrophysical Journal, 752(2):81.

Deming, D., Wilkins, A., McCullough, P., Burrows, A., Fortney, J. J., Agol, E., Dobbs-Dixon, I., Madhusudhan, N., Crouzet, N., Désert, J.-M., Gilliland, R. L., Haynes, K., Knutson, H. A., Line, M., Magic, Z., Mandell, A. M., Ranjan, S., Charbonneau, D., Clampin, M., Seager, S., and Showman, A. P. (2013) INFRARED TRANSMISSION SPECTROSCOPY OF THE EXOPLANETS HD 209458b AND XO-1b USING THE WIDE FIELD CAMERA-3 ON THE HUBBLE SPACE TELESCOPE. The Astrophysical Journal, 774(2):95

des Etangs, A. L., Vidal-Madjar, A., Desert, J. M., and Sing, D. (2008). Rayleigh scattering by H2 in the extrasolar planet HD209458b. arXiv.org.

Desert, J. M., Vidal-Madjar, A., des Etangs, A. L., Sing, D., Ehrenreich, D., Hebrard, G., and Ferlet, R. (2008). TiO and VO broad band absorption features in the optical spectrum of the atmosphere of the hot-Jupiter HD209458b. arXiv.org.

Diamond-Lowe, H., Stevenson, K., Bean, J., Line, M., and Abbot, D. (2014). A new look at the thermal inversion of hd $209458 \mathrm{~b}$.

Earl, B. L. and Herm, R. R. (1974). Photodissociation of NaBr, $\mathrm{Nal}$, and KI vapors and collisional quenching of Na. The Journal of Chemical Physics, 60(11):4568.

Fortney, J. J., Sudarsky, D., Hubeny, I., Cooper, C. S., Hubbard, W. B., Burrows, A., and Lunine, J. I. (2003). On the Indirect Detection of Sodium in the Atmosphere of the Planetary Companion to HD 209458. The Astrophysical Journal, 589(1):615-622.

Geltman, S. (1988). Semiclassical analysis of the associative ionisation process $\mathrm{Na}(3 \mathrm{p})+\mathrm{Na}(3 \mathrm{p})$ to $\mathrm{Na} 2++\mathrm{e}$. Journal Of Physics B-Atomic Molecular And Optical Physics, 21(24):L735-L740.

Geltman, S. (1989). Theory of energy-transfer collisions of excited sodium atoms: $3 \mathrm{P}+3 \mathrm{P} 3 \mathrm{~S}+5 \mathrm{~S}$ or $4 \mathrm{D}$. Physical Review A, 40(5):2301-2308.

Gilligan, J. J., McCunn, L. R., Leskiw, B. D., Herman, Z., and Castleman, Jr, A. W. (2001). Associative ionization of excited sodium species with various ligands: assessing relative bonding strengths of ion-ligand interactions. International Journal Of Mass Spectrometry, 204(1-3):247-253.

Hubbard, W. B., Fortney, J. J., Lunine, J. I., Burrows, A., Sudarsky, D., and Pinto, P. (2001). Theory of Extrasolar Giant Planet Transits. The Astrophysical Journal, 560(1):413-419.

Huennekens, J. and Gallagher, A. (1983a). Associative ionization in collisions between two $\mathrm{Na}(3 \mathrm{P})$ atoms. Physical Review A, 28(3):1276-1287.

Huennekens, J. and Gallagher, A. (1983b). Cross sections for energy transfer in collisions between two excited sodium atoms. Physical Review A, 27(2):771-784.

Husain, D. and Lee, Y. H. (1988). Measurement of absolute rate data for the reaction of atomic potassium, $\mathrm{k}(42 \mathrm{~s})$, with ch3f, c2h5f, c6h5f, ch3br, c2h5br, hcl and hbr by time-resolved atomic resonance absorption spectroscopy at $=404 \mathrm{~nm}$ $(\mathrm{k}(52 \mathrm{pj}) \mathrm{k}(42 \mathrm{~s}))$. International Journal of Chemical Kinetics, 20(3):223-240.

Husain, D. and Marshall, P. (1986). Determination of absolute rate data for the reactions of atomic sodium, na(32s1/2), with ch3f, ch3cl, ch3br, hcl, and hbr as a function of temperature by time-resolved atomic resonance spectroscopy. International Journal of Chemical Kinetics, 18(1):83-98.
Husser, T. O., Wende-von Berg, S., Dreizler, S., Homeier, D. Reiners, A., Barman, T., and Hauschildt, P. H. (2013). A new extensive library of PHOENIX stellar atmospheres and synthetic spectra. Astronomy and Astrophysics, 553:6. Iro, N., Bezard, B., and Guillot, T. (2005). A time-dependent radiative model of HD209458b. Astronomy and Astrophysics, 436(2):719-727

Jensen, A. G., Redfield, S., Endl, M., Cochran, W. D., Koesterke, L., and Barman, T. S. (2011). A SURVEY OF ALKALI LINE ABSORPTION IN EXOPLANETARY ATMOSPHERES. The Astrophysical Journal, 743(2):203.

Jensen, D. E. (1982). Alkali-metal compounds in oxygen-rich flames. A reinterpretation of experimental results. Journal of the Chemical Society, Faraday Transactions 1: Physical Chemistry in Condensed Phases, 78(9):2835.

Jensen, D. E., Jones, G. A., and Mace, A. C. H. (1979). Flame inhibition by potassium. Journal of the Chemical Society, Faraday Transactions 1: Physical Chemistry in Condensed Phases, 75(0):2377.

Kimura, M. and Saikan, S. (1981). Penning and associative ionizations in collisions of laser-excited $\mathrm{Na}$ atoms with $\mathrm{K}$ atoms. Chemical Physics Letters, 84(2):276-279.

Kleiber, P. D., Wong, T. H., and Bililign, S. (1993). Collisional energy transfer in $\mathrm{Na}(4 \mathrm{p}-3 \mathrm{~d})-\mathrm{He}, \mathrm{H} 2$ collisions. The Journal of Chemical Physics.

Klucharev, A., Sepman, V., and Vujnovic, V. (1977). Associative ionization from the resonance levels of potassium. Journal of Physics B: Atomic and Molecular Physics, 10(4):715-720.

Knutson, H. A., Charbonneau, D., Allen, L. E., Burrows, A., and Megeath, S. T. (2008). The 3.6-8.0 $\mu \mathrm{m}$ Broadband Emission Spectrum of HD 209458b: Evidence for an Atmospheric Temperature Inversion. The Astrophysical Journal, 673:526.

Knutson, H. A., Charbonneau, D., and Noyes, R. W. (2007) Using Stellar Limb-Darkening to Refine the Properties of HD $209458 \mathrm{~b}$ - Abstract - The Astrophysical Journal - IOPscience. The Astrophysical ...

Koskinen, T. T., Harris, M. J., Yelle, R. V., and Lavvas, P. (2013a). The escape of heavy atoms from the ionosphere of HD209458b. I. A photochemical - dynamical model of the thermosphere. Icarus, 226:1678-1694.

Koskinen, T. T., Lavvas, P., Yelle, R. V., and Cho, J.-K. (2014). Electrodynamics on close-in extrasolar giant planets. submitted to The Astrophysical Journal.

Koskinen, T. T., Yelle, R. V., Harris, M. J., and Lavvas, P. (2013b). The escape of heavy atoms from the ionosphere of HD209458b. II. Interpretation of the observations. Icarus, 226:1695-1708

Koskinen, T. T., Yelle, R. V., Lavvas, P., and Lewis, N. K. (2010). Characterizing the Thermosphere of HD209458b with UV Transit Observations. The Astrophysical Journal, 723:116-128.

Kramida, A., Yu. Ralchenko, Reader, J., and and NIST ASD Team (2013). NIST Atomic Spectra Database (ver. 5.1), [Online]. Available: http://physics.nist.gov/asd [2014, February 28]. National Institute of Standards and Technology, Gaithersburg, MD.

Kushawaha, V. S. and Leventhal, J. J. (1982). Associative ionization in laser-excited sodium vapor. Physical Review A (General Physics), 25(1):346-350.

Landini, M. and Fossi, B. C. M. (1991). Ion equilibrium for minor components in a thin plasma. Astronomy and Astrophysics Supplement Series (ISSN 0365-0138), 91:183-196.

Lavvas, P., Yelle, R. V., Koskinen, T., Bazin, A., Vuitton, V., Vigren, E., Galand, M., Wellbrock, A., Coates, A. J., Wahlund, J.-E., Crary, F. J., and Snowden, D. (2013). Aerosol growth in Titan's ionosphere. In Proceedings of the National Academy of Sciences, pages 2729-2734.

Lavvas, P. P., Coustenis, A., and Vardavas, I. M. (2008). Coupling photochemistry with haze formation in Titan's atmosphere, part I: Model description. Planetary And Space Science, 56(1):27-66.

Lin, K. C. and Schilowitz, A. M. (1984). Collisional deactivation of potassium (52PJ) by molecular hydrogen. Identification of the primary quenching channel. The Journal of Physical Chemistry, 88(6670-6675).

Lin, K.-C. and Vetter, R. (2002). Alkali-hydrogen reactions. International Reviews in Physical Chemistry, 21(3):357-383.

Linsky, J. L., Yang, H., France, K., Froning, C. S., Green, J. C. Stocke, J. T., and Osterman, S. N. (2010). OBSERVATIONS OF MASS LOSS FROM THE TRANSITING EXOPLANET HD 209458b. The Astrophysical Journal, 717(2):1291-1299.

Liu, D.-K. and Lin, K.-C. (1996). Rotational population distribution of $\mathrm{KH}(\mathrm{v}=0,1,2$, and 3$)$ in the reaction of $\mathrm{K}(52 \mathrm{PJ}, 62 \mathrm{PJ}$, and $72 \mathrm{PJ})$ with $\mathrm{H} 2$ : Reaction mechanism and product energy disposal. The Journal of Chemical Physics, 105(20):9121. 
Lodders, K. (2010a). Formation and Evolution of Exoplanets, chapter 8, pages 157-186. Wiley-VCH.

Lodders, K. (2010b). Solar System Abundances of the Elements.

Mayer, S. W., Schieler, L., and Johnston, H. S. (1966).

Computed High-Temperature Rate Constants for

Hydrogen-Atom Transfers Involving Light Atoms. Journal Of Chemical Physics, 45:385.

McClean, R. E., Nelson, H. H., and Campbell, M. L. (1993). Kinetics of the reaction al $\left({ }^{2} p^{0}\right)+\mathrm{h}_{2} \mathrm{O}$ over an extended temperature range. J. Phys. Chem., 97:9673-9676.

Measures, R. M. (1977). Efficient laser ionization of sodium vapor - A possible explanation based on superelastic collisions and reduced ionization potential. Journal of Applied Physics, 48:2673-2675.

Moses, J. I., Visscher, C., Fortney, J. J., Showman, A. P., Lewis, N. K., Griffith, C. A., Klippenstein, S. J., Shabram, M., Friedson, A. J., Marley, M. S., and Freedman, R. S. (2011). Disequilibrium Carbon, Oxygen, and Nitrogen Chemistry in the Atmospheres of HD 189733b and HD 209458b. The Astrophysical Journal, 737:15.

Motzkus, M., Pichler, G., and Kompa, K. L. (1997). Comparison of the $\mathrm{Na}(4 \mathrm{p})+\mathrm{H} 2$ and $\mathrm{Na}(3 \mathrm{p})+\mathrm{H} 2$ reactive/quenching systems studied with CARS, resonance-enhanced CARS, and DFWM. The Journal of Chemical ....

Motzkus, M., Pichler, G., Kompa, K. L., and Hering, P. (1998). Vibrationally induced formation of $\mathrm{NaH}$ in the $\mathrm{Na}(3 \mathrm{p})+\mathrm{H}[\mathrm{sub}$ 2] collision system: Rate equation model and comparison with experimental results. The Journal of Chemical Physics, 108(22):9291.

Muller, C. H., Schofield, K., and Steinberg, M. (1980). Laser induced flame chemistry of $\mathrm{Li}(22 \mathrm{P} 1 / 2,3 / 2)$ and $\mathrm{Na}$ $(32 \mathrm{P} 1 / 2,3 / 2)$. Implications for other saturated mode measurements. The Journal of Chemical Physics, 72(12):6620-6631.

Nahar, S. N., Bautista, M. A., and Pradhan, A. K. (1997). ElectronIon Recombination of Neutral Iron. The Astrophysical Journal, 479(1):497-503.

Namiotka, R., Huennekens, J., and Allegrini, M. (1997). Energy-pooling collisions in potassium: $4 \mathrm{P}_{J}+4 \mathrm{P}_{J} 4 \mathrm{~S}+(\mathrm{nl}=5 \mathrm{P}, 6 \mathrm{~S}, 4 \mathrm{D})$. Physical Review A, 56(1):514-520.

Parmentier, V., Showman, A. P., and Lian, Y. (2013). 3D mixing in hot Jupiters atmospheres. I. Application to the day/night cold trap in HD 209458b. Astronomy \& Astrophysics, 558:91.

Patrick, R. and Golden, D. M. (1984). Termolecular reactions of alkali metal atoms with $\mathrm{o} 2$ and oh. International Journal of Chemical Kinetics, 16(12):1567-1574.

Sandner, W., Gallagher, T., Safinya, K., and Gounand, F. (1981). Photoionization of potassium in the vicinity of the minimum in the cross section. Physical Review A, 23(5):2732-2735.

Seager, S. and Sasselov, D. D. (2000). Theoretical Transmission Spectra during Extrasolar Giant Planet Transits. The Astrophysical Journal, 537(2):916-921.

Semenov, D. and Wiebe, D. (2011). Chemical Evolution of Turbulent Protoplanetary Disks and the Solar Nebula. The Astrophysical Journal Supplement, 196(2):25.

Sharipov, A., Titova, N., and A, S. (2011). Kinetics of the al + $\mathrm{h}_{2} \mathrm{O}$ reaction: Theoretical study. J. Phys. Chem. A, 115(17):4476-4481.

Shimansky, V. V., Bikmaev, I. F., Galeev, A. I., Shimanskaya, N. N., Ivanova, D. V., Sakhibullin, N. A., Musaev, F. A., and Galazutdinov, G. A. (2003). Observational constraints on potassium synthesis during the formation of stars of the Galactic disk. Astronomy Reports, 47(9):750-762.

Shkolnik, E. L. (2013). An ultraviolet investigation of activity on exoplanet host stars. The Astrophysical Journal, 766(1):9.

Showman, A., Fortney, J., and Lian, Y. (2009). Atmospheric circulation of hot Jupiters: Coupled radiative-dynamical general circulation model simulations of HD $189733 \mathrm{~b}$ and HD 209458b. The Astrophysical Journal.

Sing, D. K., Desert, J. M., Fortney, J. J., Lecavelier des Etangs, A., Ballester, G. E., Cepa, J., Ehrenreich, D., Lopez-Morales, M., Pont, F., Shabram, M., and Vidal-Madjar, A. (2010). GTC OSIRIS Transiting Exoplanet Atmospheric Survey: Detection of potassium in XO-2b from spectrophotometry. eprint arXiv, 1008:4795.
Sing, D. K., Huitson, C. M., Lopez-Morales, M., Pont, F., Désert, J. M., Ehrenreich, D., Wilson, P. A., Ballester, G. E., Fortney, J. J., Lecavelier des Etangs, A., and Vidal-Madjar, A. (2012). GTC OSIRIS transiting exoplanet atmospheric survey: detection of sodium in XO-2b from differential long-slit spectroscopy. Monthly Notices of the Royal Astronomical Society, 426(2):1663-1670.

Sing, D. K., Vidal-Madjar, A., des Etangs, A. L., Desert, J. M., Ballester, G., and Ehrenreich, D. (2008a). Determining atmospheric conditions at the terminator of the hot-Jupiter HD209458b. arXiv.org.

Sing, D. K., Vidal-Madjar, A., Desert, J. M., Lecavelier des Etangs, A., and Ballester, G. (2008b). Hubble Space Telescope STIS Optical Transit Transmission Spectra of the Hot Jupiter HD 209458b. The Astrophysical Journal, 686(1):658-666.

Snellen, I. A. G., Albrecht, S., de Mooij, E. J. W., and Le Poole, R. S. (2008). Ground-based detection of sodium in the transmission spectrum of exoplanet HD209458b. Astronomy and Astrophysics, 487(1):357-362.

Su, W., Ji-Ping, C., Yu-Zhong, H., Bing-Cheng, F., and Jing, W. (2001). Shock Tube Studies on Recombination Kinetics of Sodium Ion with Electron. Chinese Physics Letters, 18(2):289-291.

Swain, M. R., Tinetti, G., Vasisht, G., Deroo, P., Griffith, C., Bouwman, J., Chen, P., Yung, Y., Burrows, A., Brown, L. R. Matthews, J., Rowe, J.'F. Kuschnig, R., and Angerhausen, D. (2009). WATER, METHANE, AND CARBON DIOXIDE PRESENT IN THE DAYSIDE SPECTRUM OF THE EXOPLANET HD 209458b. Astrophysical Journal, 704(2):1616-1621.

Swihart, M. T., L, C., Legrand, B., Gokalp, I., and Paillard, C. (2003). Rate constants for the homogenous gas-phase al/hcl combustion chemistry. Combust. Flame, 132:91-101.

Takeda, Y., Kang, D.-I., Han, I., Lee, B.-C., Kim, K.-M., Kawanomoto, S., and Ohishi, N. (2012). Lithium, Sodium, and Potassium Abundances in Sharp-Lined A-Type Stars Takeda, Yoichi; Kang, Dong-Il; Han, Inwoo; Lee, Byeong-Cheol; Kim, Kang-Min; Kawanomoto, Satoshi; Ohishi, Naoko;. Publications of the Astronomical Society of Japan, 64:38.

Venot, O., Hébrard, E., Agúndez, M., Dobrijevic, M., Selsis, F., Hersant, F., Iro, N., and Bounaceur, R. (2012). A chemical model for the atmosphere of hot Jupiters. Astronomy and Astrophysics, 546:A43.

Verner, D. A., Ferland, G. J., Korista, K. T., and Yakovlev, D. G. (1996). Atomic data for astrophysics. ii. new analytic fits for photoionization cross sections of atoms and ions. The Astrophysical Journal, 465(487-498).

Vidal-Madjar, A., des Etangs, A., and Désert, J. (2003). An extended upper atmosphere around the extrasolar planet HD209458b. Nature, 422:143-146.

Vidal-Madjar, A., Désert, J.-M., Lecavelier des Etangs, A., Hébrard, G., Ballester, G. E., Ehrenreich, D., Ferlet, R. McConnell, J. C., Mayor, M., and Parkinson, C. D. (2004) Detection of Oxygen and Carbon in the Hydrodynamically Escaping Atmosphere of the Extrasolar Planet HD $209458 \mathrm{~b}$. The Astrophysical Journal, 604:L69.

Vidal-Madjar, A., Sing, D. K., Lecavelier des Etangs, A., Ferlet, R., Desert, J. M., Hebrard, G., Boisse, I., Ehrenreich, D., and Moutou, C. (2011). The upper atmosphere of the exoplanet HD209458b revealed by the sodium D lines. Astronomy and Astrophysics, 527:A110.

Weiss, P. S., Mestdagh, J. M., Covinsky, M. H., and Balko, B. A. (1988). The reactions of ground and excited state sodium atoms with hydrogen halide molecules. Chemical Physics, 126(93-109).

Wippel, V., Binder, C., Huber, W., Windholz, L., Allegrini, M., Fuso, F., and Arimondo, E. (2001). Photoionization cross-sections of the first excited states of sodium and lithium in a magneto-optical trap. The European Physical Journal D, 17(3):285-291.

Wong, T., PD, K., and KH, Y. (1999). Chemical dynamics of the reaction $\mathrm{K}^{*}(5 \mathrm{p}(2) \mathrm{P})+\mathrm{H}-2-i \mathrm{KH}(\mathrm{v}=0 ; \mathrm{J})+\mathrm{H}$ : Electronic orbital alignment effects. Journal Of Chemical Physics, 110(14):6743-6748.

Xie, J.-W., Brandeker, A., and Wu, Y. (2013). ON THE UNUSUAL GAS COMPOSITION IN THE $\beta$ PICTORIS DEBRIS DISK. The Astrophysical Journal, 762(2):114.

Zahnle, K., Marley, M. S., Freedman, R. S., Lodders, K., and Fortney, J. J. (2009). Atmospheric Sulfur Photochemistry on Hot Jupiters. The Astrophysical Journal Letters, 701(1):L20-L24.

Zatsarinny, O. and Tayal, S. S. (2010). Photoionization of potassium atoms from the ground and excited states. Physical Review A, 81(4):043423.

Zhang, Z. and Shneider, M. N. (2010). Measurement of plasma decay processes in mixture of sodium and argon by coherent microwave scattering. Physics of Plasmas, 17(3):033108. 